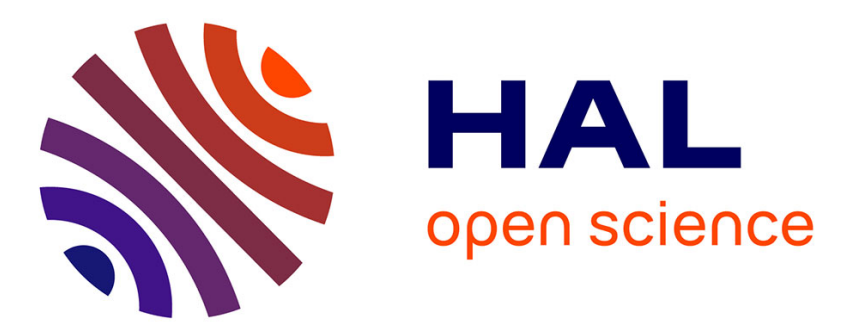

\title{
Electrophysiological monitoring of inhibition in mammalian species, from rodents to humans
}

\author{
Adrien Peyrache, Alain Destexhe
}

\section{To cite this version:}

Adrien Peyrache, Alain Destexhe. Electrophysiological monitoring of inhibition in mammalian species, from rodents to humans. Neurobiology of Disease, 2019, 130, pp.104500. 10.1016/j.nbd.2019.104500 . hal-02157942

\section{HAL Id: hal-02157942 \\ https://hal.science/hal-02157942}

Submitted on 17 Jun 2019

HAL is a multi-disciplinary open access archive for the deposit and dissemination of scientific research documents, whether they are published or not. The documents may come from teaching and research institutions in France or abroad, or from public or private research centers.
L'archive ouverte pluridisciplinaire $\mathbf{H A L}$, est destinée au dépôt et à la diffusion de documents scientifiques de niveau recherche, publiés ou non, émanant des établissements d'enseignement et de recherche français ou étrangers, des laboratoires publics ou privés. 


\title{
Electrophysiological monitoring of inhibition in mammalian species, from rodents to humans.
}

\author{
Adrien Peyrache ${ }^{1, *}$ and Alain Destexhe ${ }^{2}$ \\ 1 Montreal Neurological Institute, McGill University, 3801 University Street, \\ Montreal, QC, H3A 2B4 Canada \\ ${ }^{2}$ Unité de Neurosciences, Information, et Complexité, Centre National de la \\ Recherche Scientifique, 91198 Gif-sur-Yvette, France \\ * To whom correspondence should be addressed: adrien.peyrache@mcgill.ca
}

\section{Abstract}

GABAergic interneurons constitute a highly diverse family of neurons that play a critical role in cortical functions. Due to their prominent role in cortical network dynamics, genetic, developmental, or other dysfunctions in GABAergic neurons have been linked to neurological disorders such as epilepsy. Thus it is crucial to investigate the interaction of these various neurons and to develop methods to specifically and directly monitor inhibitory activity in vivo. While research in small mammals has benefited from a wealth of recent technological development, bridging the gap to large mammals and humans remains a challenge. This is of particular interest since single neuron monitoring with intracranial electrodes in epileptic patients is developing quickly, opening new avenues for understanding the role of different cell types in epilepsy. Here we review currently available techniques that monitor inhibitory activity in the brain and the respective validations in rodents. Finally, we discuss the future developments of these techniques and how knowledge from animal research can be translated to the study of neuronal circuit dynamics in the human brain.

\section{Outline.}

Electrophysiological monitoring of cortical inhibition in mammalian species, from rodents to humans..................................................................................................................... 1

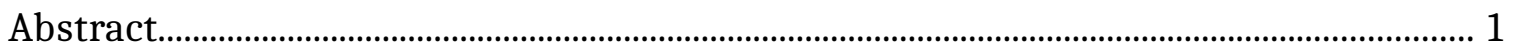

Outline

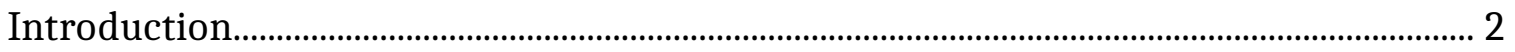

Diversity of neuronal cell types in the brain..........................................................................

Electrophysiological markers of inhibitory activity............................................................... 5 
Fast-spiking neurons are inhibitory.............................................................................. 5

Fast-spiking neurons have distinct extracellular waveforms............................................6

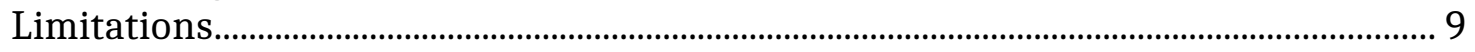

Inter-neuronal markers of cell types..................................................................................... 10

Putative synaptic connections............................................................................................. 10

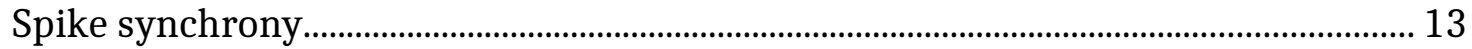

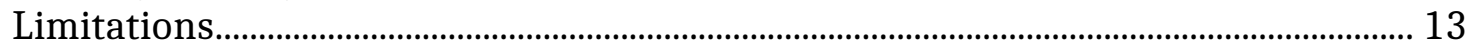

Other extracellular markers of cell types.................................................................................. 14

Anatomical distribution..................................................................................................... 14

Oscillatory modulation................................................................................................... 14

Waveforms from inhibitory pre-synaptic terminals..........................................................15

Population inhibitory post-synaptic potentials............................................................... 15

Electrophysiological markers of inhibitory activity are potentially universal..............16

Characterizing cell types in NHPs and humans.............................................................19

Oscillatory markers of cell types............................................................................................ 19

Properties and role of inhibitory networks..........................................................................21

Generation of action potentials........................................................................................ 21

Long-range coordination of brain activity.....................................................................22

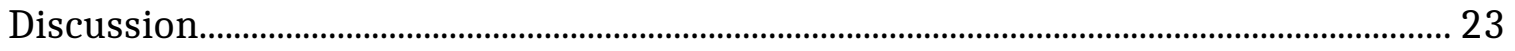

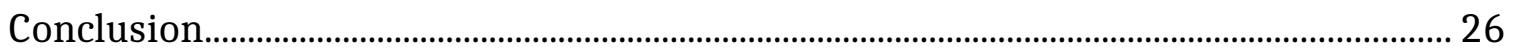

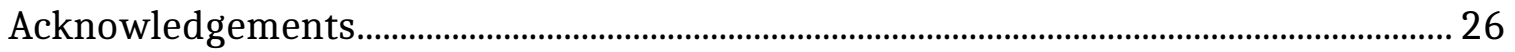

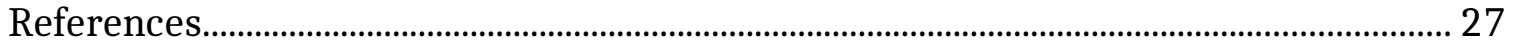

\section{Introduction}

The observation that neurons in the brain are highly diverse dates back to the seminal anatomical studies of Ramon y Cajal and Lorente de No. A century of intense research in this field has demonstrated that cortical neurons can be broadly divided into two families based on the neurotransmitter they release: glutamatergic and GABAergic neurons which depolarize and hyperpolarize post-synaptic targets, respectively, at resting membrane potentials (Connors and Gutnick, 1990; McCormick et al., 1985; Zeng and Sanes, 2017). The interplay between the various classes of cells results in rich cortical dynamics, which span orders of magnitude in timescale (Buzsaki and Draguhn, 2004). Inhibitory neurons play various roles in ongoing brain dynamics and are crucial in the operation of cortical circuits (Hattori et al., 2017; Isaacson and Scanziani, 2011; Kepecs and Fishell, 2014; Roux and Buzsáki, 2015; Wood et al., 2017).

Inhibition enables the temporal organization of spiking activity in patterns that can be detected by extracranial sensors (with Electro-Encephalography, EEG, or magneto-encephalography MEG) or intracranial electrodes (Local Field Potentials, LFP) (Buzsáki et al., 2012). Brain rhythms thus reflect the dynamic interaction of large constellations of neurons, that enable coordination and information transmission within local and global neuronal networks (Buzsaki and Draguhn, 
2004; Fries, 2005). Neuronal dynamics play such a fundamental role that any perturbation can potentially lead to debilitating cognitive disorders, or serve as biomarkers of these disorders (Buzsáki and Watson, 2012; Ferrarelli et al., 2007; Fitzgerald and Watson, 2018). In particular, defects in the maturation of the inhibitory networks can lead to severe neurological disorders (Marín, 2012). The central role of inhibitory malfunction is perhaps most well studied in the context of epilepsy (Avoli et al., 2016; Goldberg and Coulter, 2013; Grasse et al., 2013; Lévesque et al., 2016; Neumann et al., 2017). This imbalance between excitation and inhibition is associated with particular inter-ictal events and high frequency oscillations that constitute promising candidates for anatomical localization of the epilepsy locus (Zijlmans et al., 2012).

Although brain oscillations at the level of the scalp reflect the interaction between excitatory and inhibitory neocortical neurons, it is impossible to separate the contributions of the respective cell classes. While excitatory neurons show fairly homogeneous morphological and dynamic features, inhibitory neurons are highly diverse in nature (Freund and Buzsáki, 1996; Rudy et al., 2011; Tremblay et al., 2016). Inhibitory neurons can be separated into three categories based on gene expression, origin of inputs, and axonal targets. More detailed analyses show that inhibitory neurons can be sub-divided into many more classes (Freund and Buzsáki, 1996; Harris et al., 2018; Rudy et al., 2011; Tasic et al., 2018; Tremblay et al., 2016), thus raising the question of whether particular cell types, or inhibitory cells in general, play a specific role in neuronal disorders (Marín, 2012; Medrihan et al., 2017).

The vast diversity of inhibitory neurons adds a level of complexity to the neurophysiology of brain dynamics thus making it difficult to study EEG or LFP alone. Fortunately, various methods are available to monitor the activity of inhibitory neurons at various scales. These methods can demonstrate the total inhibitory current received by a single neuron in in vivo whole-cell patch clamp preparation (Borg-Graham et al., 1998; Wehr and Zador, 2003) to the simultaneous activity of multiple inhibitory neurons (Fujisawa et al., 2008; Stark et al., 2013; Wilson et al., 2012). Most of these techniques are well fitted for small animal research, especially mice, due to a large genetic targeting toolbox already available (Taniguchi et al., 2011). This also allows for the cell-class specific monitoring of inhibitory neurons with optical imaging techniques (e.g. Kerlin et al., 2010; Pinto and Dan, 2015). However, these methods fail to translate to other animals such as primates, and even less to humans. Despite this, recent technological developments allow for the monitoring of inhibitory activity in human subjects. Micro-electrode recordings in human epileptic patients (Babb et al., 1987; Cash and Hochberg, 2015; Engel et al., 2005) allow for the detection of inhibitory activity at the resolution of single neurons (Peyrache et al., 2012; Le Van Quyen et al., 2008; Weiss et al., 2015). Non-invasive techniques for monitoring inhibition are also available, such as transcranial magnetic stimulation (Kujirai et al., 1993) and magnetic resonance spectroscopy (Puts and Edden, 2012). 
We will first briefly review how cortical inhibitory interneurons can be classified based on various genomic, morphological, and circuit integration features. We will then review currently available techniques that monitor inhibitory activity in animal research, especially in small rodents. Finally, we will see how inhibition can be monitored in humans, sometimes with techniques translated from animal research. We will then conclude that a successful diagnostic of inhibitory malfunction in the neocortex, especially in epilepsy, can best be addressed by a combination of complementary techniques.

\section{Diversity of neuronal cell types in the brain}

Cortical neurons are split into two categories depending on whether they release glutamate or Gamma-Aminobutyric Acid (GABA) at their presynaptic terminals (Connors and Gutnick, 1990; Zeng and Sanes, 2017). Glutamatergic cells are, in large majority, pyramidal-shaped neurons that depolarize their post-synaptic targets. Contrastingly, GABAergic neurons hyperpolarize and inhibit post-synaptic neurons. Although they represent a minority of the cortical neurons, approximatively $20 \%$, they show exceptional diversity in genomic expression, morphology, synaptic inputs, and axonal targeting (Freund and Buzsáki, 1996; Rudy et al., 2011; Tremblay et al., 2016). The most studied classes are certainly basket cells, which control somatic inhibition, and Martinotti cells, which target distal apical dendrites of pyramidal neurons (Freund and Buzsáki, 1996; Kepecs and Fishell, 2014; Somogyi and Klausberger, 2005; Tremblay et al., 2016). Each of these GABAergic cell types populates cortical layers differentially, and the anatomical distribution is preserved across cortical structures (Tremblay et al., 2016; Xu et al., 2010).

Recently, GABAergic neurons have been classified by electrophysiological characteristics and molecular markers such as gene expression patterns. They can be divided into three large families, each amounting to approximately the same number of neurons. These properties have been extensively reviewed in literature (Freund and Buzsáki, 1996; Rudy et al., 2011; Tremblay et al., 2016) and will be only briefly discussed here. These three families can be characterized by single genetic markers: parvalbumin (PV), somatostatin (SST), and 5-hydroxytryptamine 3a receptors (5HT3AR). Other classifications divide the latter category into two classes of GABAergic neurons that either express the vasoactive intestinal peptide (VIP) or reelin (Wamsley and Fishell, 2017). In the former class, the VIP-positive neurons have been the most studied in vivo since specific Cre-driver mouse lines are now available (Taniguchi et al., 2011).

The three families of GABAergic neurons manage different functions in cortical circuits. For example, PV-positive neurons can be either soma-targeting basket cells or axonal-targeting chandelier cells and thereby can control the output of the pyramidal neurons. PV-positive basket cells exert a sustained inhibitory drive to targeted pyramidal neurons. They also receive direct thalamic inputs which allow temporally precise sensory responses in the cortex through feed forward inhibition 
to pyramidal cells (Alonso and Swadlow, 2005; Cruikshank et al., 2007; Gabernet et al., 2005). In contrast, SST-positive neurons including Martinotti cells, target pyramidal cell dendrites, thus controlling the input of the circuit and providing feedback to local pyramidal cells (Kvitsiani et al., 2013; Silberberg and Markram, 2007; Simonnet et al., 2017). In addition, SST-positive neurons are mainly driven by local excitatory cells and are weakly driven by thalamic inputs (Cruikshank et al., 2010; Nassar et al., 2018), although they may be targeted by thalamocortical neurons (Ährlund-Richter et al., 2019). Finally, VIP positive neurons form disinhibitory circuits that principally target other inhibitory neurons (Pi et al., 2013; Yu et al., 2019).

\section{Electrophysiological markers of inhibitory activity}

Understanding the role of GABAergic neurons in cortical function require tools to monitor these neurons. Historically, extracellular electrophysiology has been the technique of choice for monitoring neuronal activity at the single cell resolution in vivo. This is also one of the only techniques, to date, that can be translated from animals to humans. In particular, human epileptic patients suffering from drugresistant forms of epilepsy can be implanted with intracerebral electrodes to localize the epileptic zone. Extracellular recordings give access to several markers of cell types, enabling the monitoring of specific GABAergic neurons. These markers were characterized and validated with a combination of methods, such as intracellular recordings and optogenetic-labeling of neurons.

\section{Fast-spiking neurons are inhibitory}

Intracellular recordings of neurons in vitro allow for the parallel investigation of electrical properties of neurons, their morphology (by filling their cell body with a fluorescent dye) and, to some extent, their genetic expression pattern. For a long time, these investigations have focused on the properties of pyramidal cells as they form the majority of cortical neurons and have large, and thus accessible, somatas. McCormick and collaborators (1985) performed the first intracellular recordings of GABAergic neurons, and they thus described two key properties: first, all fastspiking (FS) cells, that had previously been described, are in fact GABAergic neurons; second, FS neurons exhibit short-duration action potentials. In particular, FS neurons show fast repolarization (potassium) currents, that enable them to fire at high rates.

\section{Extracellular properties of different cell classes.}


For each individual neuron recorded extracellularly, two main properties are immediately available: the waveform of the extracellular action potentials and the dynamics of the spike train emission. These two properties can be used to determine the cell type.

\section{Bimodal distribution of extracellular waveforms}

Extracellular electrical waveforms vary from neuron to neuron due to different intracellular properties and the position of the electrodes relative to the soma (Gold, 2006). The negative temporal derivative of the membrane potential can be used to approximate extracellular potentials associated with the generation of an action potential (Gold et al., 2006). Hence, the rising and falling phase of the action potential leads to a bi- or tri-phasic extracellular waveform characterized by a trough (maximum depolarization rate) followed by a peak (maximum repolarization current rate). A waveform can be characterized by two intrinsic values: the width of the peak and the trough-to-peak duration (Figure 1A). While waveforms have been characterized using other features, for example spike waveform asymmetry (Sirota et al., 2008) or overall spike duration (Stark et al., 2013), they are generally equivalent to the aforementioned features. Two of these four waveform characteristics are usually sufficient to classify the waveforms between a group of narrow and broad waveform neurons since they form a bimodal distribution of points (Figure 1B). The classification itself can be achieved by setting thresholds validated by recording cell-type specific neurons (see Table 1), or by using unsupervised classifiers such as k-means.

\section{Validation of waveform-based classification}

What classes of interneurons show narrow waveforms? Recent technology has allowed researchers to specifically label interneuron types (Taniguchi et al., 2011) Using transgenic mice, specific cells can be labeled with bioengineered opsins which cause the labeled cell type to respond to light stimulation. These optogenetic labeling techniques have thus confirmed in vivo that most PV-positive neurons have narrow waveforms (Cardin et al., 2009; Kvitsiani et al., 2013; Royer et al., 2012) (Figure 2D,E). Narrow waveform neurons cell class will thus be interchangeably referred to as FS cells. In contrast, broad waveform (BW) neurons will be referred to as putative pyramidal only when other markers are available.

Using juxtacellular recordings, combined with immunostaining and optogenetic, $\mathrm{Yu}$ and collaborators (2019) were able to obtain a dataset of waveforms specific to each cell class. While FS/PV neurons are well separated from putative pyramidal cells, the two other main inhibitory cell classes, namely SST and VIP positive neurons, show intermediate waveform durations (Figure 2C), as suggested in previous studies (e.g. Gentet et al., 2010). 
Juxtacellular recordings provide very clean waveforms while extracellular recordings are necessarily contaminated with noise, both from physiological and external origin. This results in a broader distribution of waveforms. Yet, optogenetically-labeled neurons in extracellular recordings confirm the clear separation between FS/PV neurons and the others, while SST-positive neurons overlap with other BW neurons, (Figure 2E).

A
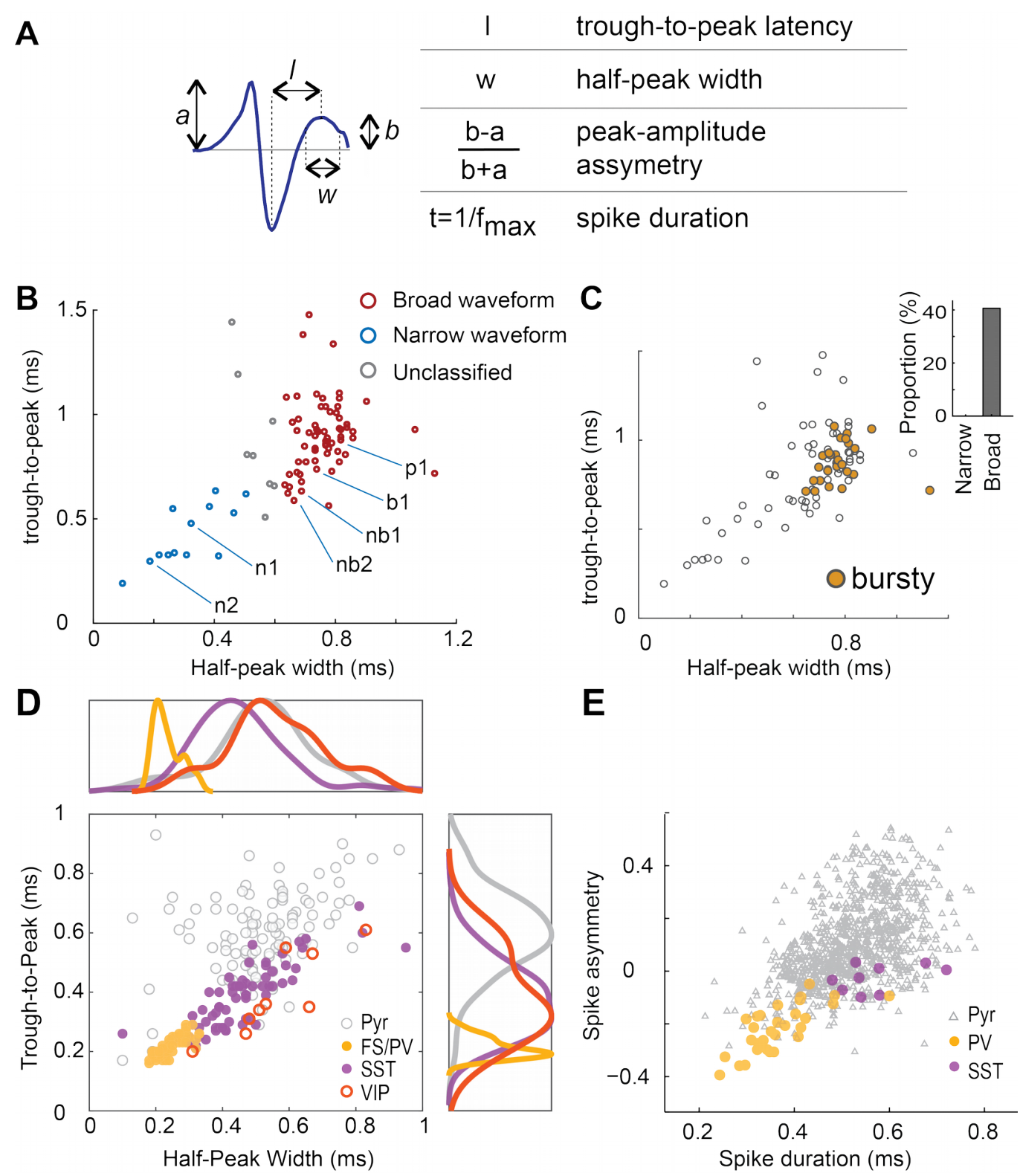

E

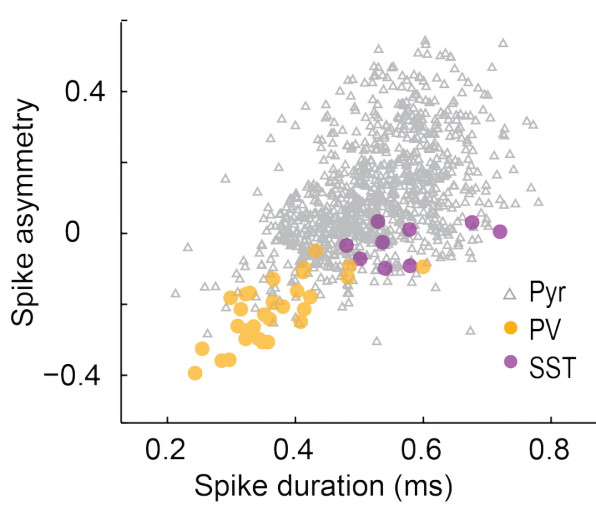

Figure 1 - Waveform-based classification of neuronal types. A. Extracellular waveform features used for cell classification. $f_{\max }$ is the frequency corresponding to maximum power in the Fourier transform of the waveform. B. Hippocampal neurons in vivo were classified by their waveform characteristics (trough-to-peak and half-width peak). The joint distribution of these two features separated broad from narrow waveforms. $\boldsymbol{C}$. Same distribution as in $\mathbf{B}$, now showing bursting neurons. Inset displays the proportion 
of broad and narrow waveform neurons categorized as 'bursty'. D. Immunostaining and optogenetic validation of cell classification in juxtacellular recordings in the barrel cortex. Juxtacellular recordings allow for a precise detection and isolation of spikes. Pyramidal (Pyr) and FS neurons were identified on waveform features (including ratio of peak to trough amplitude, not shown here, see also main text). A subset of FS neurons was identified as PV-positive by immunostaining or optogenetic labeling. SST and VIP neurons were all identified with optogenetic labeling. SST and VIP neurons show an intermediate position between FS and pyramidal cells. Note that relative density of cell types does not reflect actual distribution (inhibitory neurons are overrepresented). $\boldsymbol{E}$. Waveform distribution from extracellular recordings in the hippocampus. PV- and SST-positive neurons were labeled with optogenetics. $\boldsymbol{A}$ is adapted with permission from (Sirota et al., 2008); $\boldsymbol{B}$ and $\boldsymbol{C}$ are adapted with permission from (Medrihan et al., 2017); $\boldsymbol{D}$ is adapted with permission from (Yu et al., 2019); $\boldsymbol{E}$ is adapted with permission from (Royer et al., 2012).

\section{A variety of spike train dynamics}

The dynamics of spike train emission reflects some of the intrinsic properties of the neurons, from various levels of conductances to their morphology. These dynamics are well captured by the temporal auto-correlation functions (or 'autocorrelograms') which quantify the average spiking rate at various time-lags after a spike is emitted (Moore et al., 1970; Perkel et al., 1967). Notably, all neurons show a dip in their autocorrelogram around time 0 , reflecting the refractory period. Intrinsic dynamics of the neurons, and thus their autocorrelograms, can potentially contribute to the classification of neuronal cell type (Figure 3). For example, the burst emission (volleys of spikes emitted at a rate above 100-150 Hz), associated with a peak in the autocorrelogram below 10ms (as shown for neuron "b1" in Figure 1B,C and 2) provides important insight on the identity of the neuron. In the cortex, only excitatory neurons are bursty (Connors and Gutnick, 1990). Finer analysis of spike train auto-correlation at short timescales $(<100 \mathrm{~ms})$ reveals putative pyramidal cells with 'peaky' autocorrelograms (only mildly bursty but highly-correlated at short timescales. e.g. 'p1' in Figure 2). In contrast, narrow waveform neurons show longer refractory periods and no burst ('n1' and 'n2' in Figure 2). However, it is clear that other neurons classified as BW cells ('nb1' and 'nb2') have strikingly different dynamics than other BW neurons. It is possible that these are non-FS inhibitory neurons with intermediate waveforms (i.e. putative SST or VIP neurons). 

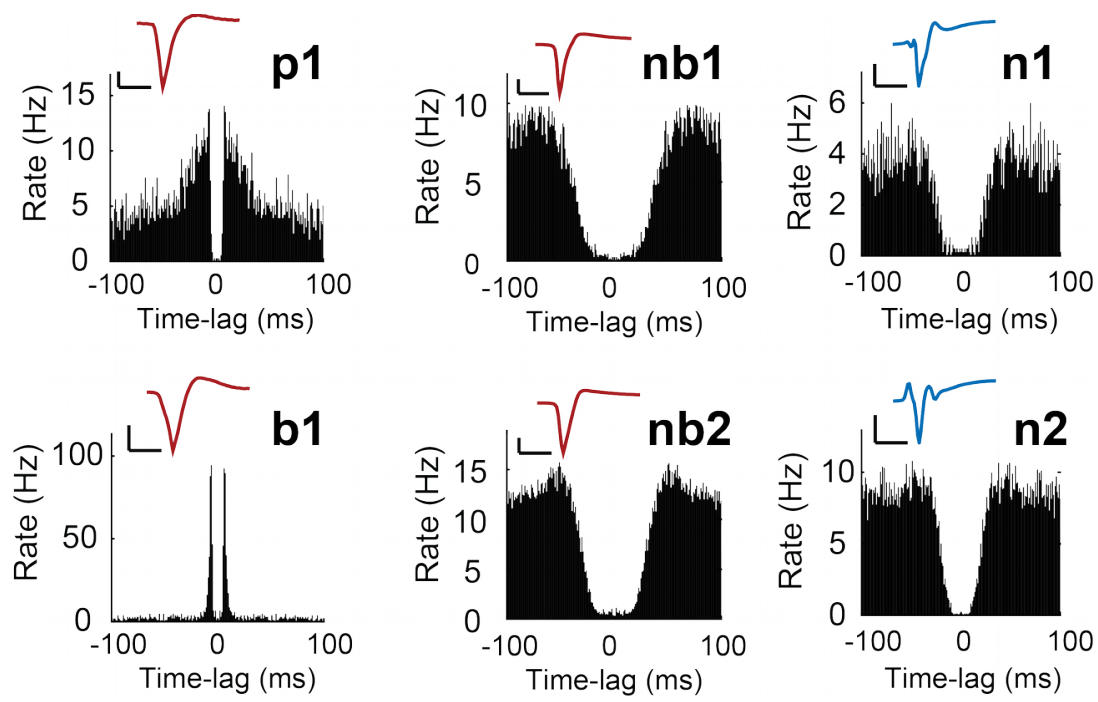

Figure 2 - Relationship between spike waveform, putative cell type and spiking dynamics. Spike train autocorrelograms and waveforms of six example neurons shown in Figure 1B. p1, peaky (but non-bursty) autocorrelogram, wide waveform neuron; b1, bursty wide waveform neuron; nb1-2, non-bursty wide waveform neurons; n1-2, narrow waveform neurons. Horizontal and vertical scale bars represent $1 \mathrm{~ms}$ and $25 \mu \mathrm{V}$, respectively. Adapted with permission from (Medrihan et al., 2017).

\section{Limitations}

While it has now been established that narrow waveform neurons are with high probability PV-positive neurons (Cardin et al., 2009; Royer et al., 2012), a full classification of neurons based on the broad range of neuronal properties available in extracellular recordings (e.g. waveforms and spike auto-correlograms) is still missing. It is becoming clear that SST and VIP-positive interneurons have 'intermediate' waveform duration. (Figure 1). Their intrinsic dynamics, as reflected by their firing rates, refractory period and other parameters (see Table 1) seem to be potential markers allowing for a fine-grained classification of inhibitory cell types in extracellular recordings but this will require further validation across cortical areas and brain states.,

Extracellular waveform features are usually bimodal since SST and VIP neurons are certainly under-sampled in most extracellular recordings (because of different anatomical distribution, among other reasons). Based on the validated juxtacellular recording datasets (Yu et al., 2019, Figure 1D), it can be established that FS/PV neurons are well separated from excitatory neurons, as shown by the large sensitivity index d' obtained from the two distributions (Table 1). Classifying SST and VIP neurons is more limited because of their intermediate distribution of waveform features. Trough-to-peak gives higher d' to separate SST and VIP from excitatory neurons. However, half-peak width is more informative when it comes to 
separate SST and VIP inhibitory neurons from FS/PV cells. The ratio of trough and peak amplitude is also often used, especially with juxtacellular recordings (e.g. Yu et al., 2019). However, how these differences in amplitude are reflected in extracellular recordings remains unclear.

The bimodal distribution of waveform features remains a tool of choice for the classification of FS/PV neurons and BW, putative pyramidal cells. One can ask how much error is made with such categorization. Assuming that $80 \%$ of cortical neurons are pyramidal and that approximately $40 \%$ of inhibitory neurons have narrow waveforms (i.e. the percentage of PV-positive neurons) (Tremblay et al., 2016), then at least $87 \%$ of broad waveform neurons are, in fact, pyramidal. Of course, this simple calculation does not account for erroneous classification that may arise from the spike detection process and waveform feature extraction.

Finally, it is to be noted that waveforms may also be incorrectly classified because of intrinsic limitations of extracellular recordings. First, the filtering procedures used to detect candidate action potentials in extracellular recordings may lead to distorted spike waveforms (Quiroga, 2009). In addition, spikes "broaden" with increasing distance from the soma, due to low-pass filtering properties of cell membrane (Gold, 2006) and extracellular space (Bédard et al., 2004). However, the first phase ( 1 millisecond) of the extracellular action potential near the soma aligns with theoretical values (Anastassiou et al., 2015), suggesting that these distortions certainly remain limited.

\section{Inter-neuronal markers of cell types}

Neurons interact at various timescales in the brain. At short time scales $(<10 \mathrm{~ms})$, extracellular spike trains show various correlations that result from synaptic and other types of fast coordination (Bartho, 2004; English et al., 2014; Moore et al., 1970; Ostojic et al., 2009). GABAergic neurons should show signs of inhibition on their post-synaptic targets and should be activated by pre-synaptic pyramidal neurons. Extracellular electrophysiology does not generally allow for the assessment of synaptic contacts between neurons, but indirect markers are available.

\section{Putative synaptic connections}

Similar to auto-correlograms, temporal cross-correlation functions (or 'crosscorrelograms') quantify the temporal relationship between reference and target spike trains at various timescales (Moore et al., 1970; Perkel et al., 1967). The crosscorrelogram is usually displayed as the average firing rate of the target cell relative to the spikes of the reference cell (i.e. the expected number of spikes in a given time window as a function of the time before or after the spikes of the reference cell). At short timescales $(\sim 1 \mathrm{~ms})$, pairwise crosscorrelograms sometimes show a shortlatency "peak" $(<5 \mathrm{~ms})$ which indicates that the spike of one neuron tends to be 
followed by the spikes of another neuron with latency compatible with synaptic transmission and post-synaptic depolarization (Bartho, 2004; English et al., 2014; Moore et al., 1970). If the peak is at positive time-lag in the cross-correlogram, the reference neuron is putatively excitatory (e.g. cell \#2 excites cell \#1 in Figure 3A). Conversely, a "dip" in the cross-correlation may indicate that one neuron inhibits the other. (e.g. cell \#1 inhibits neurons \#3 and \#4 in Figure 3A).

The short-latency and short-lasting events in the cross-correlograms may thus allow for the distinction between putative excitatory and inhibitory neurons. Crosscorrelograms computed with shuffled spike times can be used to calculate the significance of these deviations from the expected data for un-connected neurons. (Amarasingham et al., 2011; Bartho, 2004; Moore et al., 1970). This control results in null distribution of cross-correlograms in which the short-lasting events are filtered out. Then, any point that lies above or below a given percentile of the null distribution at short-latency $(<5 \mathrm{~ms})$ can be interpreted as a sign of a putative excitatory or inhibitory synaptic contact, respectively. Performing such analyses on ensembles of simultaneously recorded neurons allows for the labeling of each neuron as putatively excitatory or inhibitory. These labels match waveform-based classification with high fidelity (Bartho, 2004; Sirota et al., 2008) (Figure 3). 


\section{A}
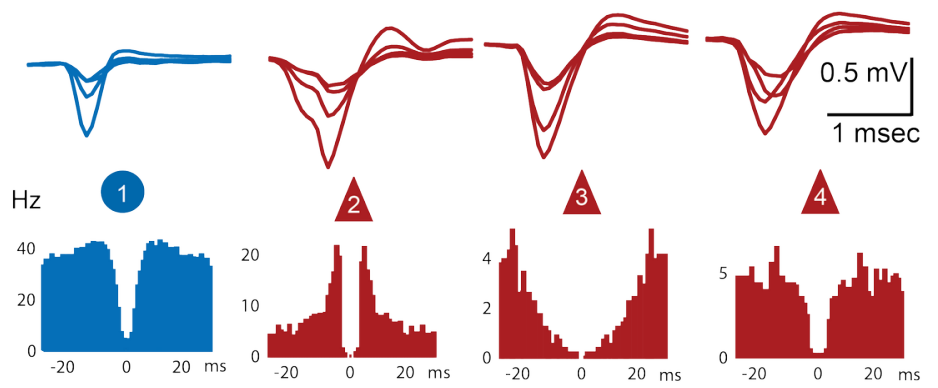

3

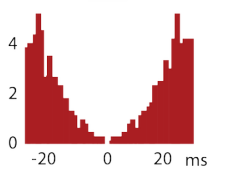

4

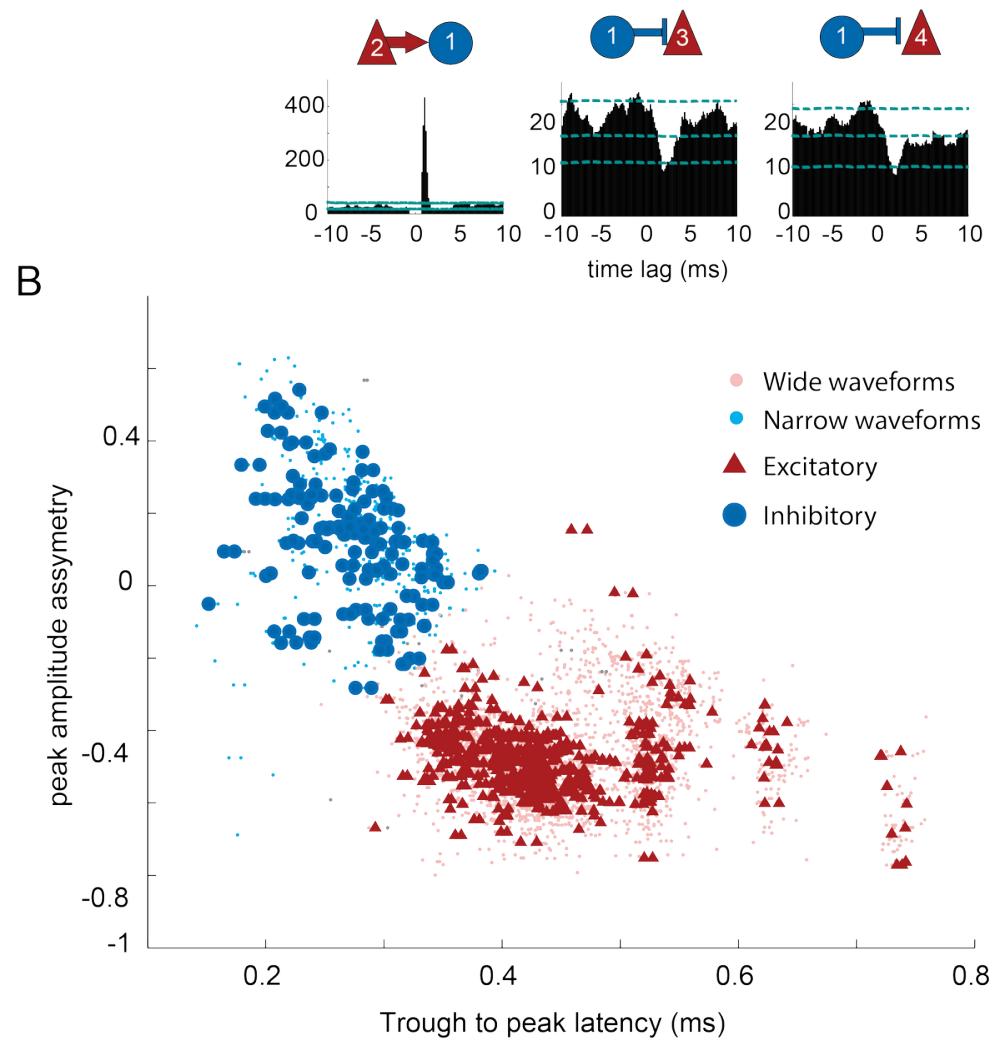

Figure 3 - Validation of cell type with identification of putative post-synaptic effect. Average wide band-recorded waveforms (1 Hz-5 kHz; upper row) and autocorrelograms (middle row) of four example units. Superimposed traces were recorded by the four tetrode sites. Bottom row, short-latency monosynaptic interactions between neuron pairs, as revealed from the cross-correlograms. Neuron 2 excites neuron 1 (recorded on the same electrode), which in turn, inhibits neurons 3 and 4 (on a different electrode). Lines indicate mean (middle), and 1\% (bottom) and 99\% (top) confidence intervals. (B) Neurons were clustered according to waveform asymmetry and mean filtered spike width (see Figure 1). Each symbol corresponds to an isolated unit ( $n=2716$, neurons recorded from the sensory and medial prefrontal cortex). Putative excitatory and inhibitory neurons form separate clusters. Circles/triangles correspond to inhibitory and excitatory neurons identified by monosynaptic interactions. Adapted with permission from Sirota et al., 2008. 


\section{Millisecond timescale spike synchrony}

Spike train synchrony is another potential marker of cell identity. Unlike putative synaptic excitation/inhibition of post-synaptic targets, cross-correlograms sometimes show a symmetric peak at 0-lag with a jitter of about 5-10 ms. In the hippocampus, synchronous spiking is frequently observed between putative interneurons, but only rarely between pyramidal cells (Diba et al., 2014). Several mechanisms have been proposed to explain millisecond synchronous spiking. One prominent hypothesis is that inter-neuronal coupling by gap junctions (electrical synapses) leads to such synchrony (Traub et al., 2001). This is potentially interesting for cell classification as gap junctions connect only interneurons of the same class (Galarreta et al., 2004; Gibson et al., 1999; Tamás et al., 2000).

PV neurons tend to be synchronous with each other, but not pairs of SST neurons (Kvitsiani et al., 2013). However, synchronous spiking is also observed between interneurons of different classes (Diba et al., 2014; Hu et al., 2011). Spiking synchrony could be achieved by synchronous inputs, for example from the thalamus (Peyrache et al., 2015), but this would require highly similar intracellular dynamic properties of the post-synaptic targets and comparable conduction delays. Postinhibitory rebound is another candidate mechanism (Hu et al., 2011). Further investigations are required to determine which neurons are synchronized and the underlying mechanisms of such phenomenon. This will open new avenues for the identification of cell class from extracellular recordings.

\section{Limitations}

Several limitations, which have already been well documented, should be considered in the analysis of cross-correlograms (Amarasingham et al., 2011; English et al., 2017; Ostojic et al., 2009; Perkel et al., 1967; Stark and Abeles, 2009). Crosscorrelograms at short timescales must be examined with caution as they result not only from putative synaptic contacts, but also from intrinsic neuronal dynamics (Ostojic et al., 2009). For example, cells' refractory period can generate a dip in negative lags of cross-correlograms, which might be incorrectly interpreted as inhibition (Ostojic et al., 2009) (see Figure 4B).

Another limitation of cross-correlogram analysis is the bias introduced during spike detection and sorting. A majority of synaptic contacts are found between nearby neurons. In other words, most putatively connected neurons can be detected on the same electrode (English et al., 2017; Fujisawa et al., 2008), thus raising issues with spike sorting. Until recently, temporally overlapping spikes could not be resolved using a given set of electrodes (tetrodes, octrodes, etc.) (Harris et al., 2000). In addition, large amplitude spikes could potentially overshadow low-amplitude ones. Recent development of a new spike sorting algorithm for multi-electrode arrays has partially resolved the issues associated with temporally overlapping spikes, on the 
condition that these spike waveforms are sufficiently different on the detection electrode (Lefebvre et al., 2016; Pachitariu et al., 2016).

\section{Other extracellular markers of inhibition and cell types}

Anatomical location and entrainment to local oscillations can be gathered from electrophysiological signals and used to assess neuronal cell type. This is particularly useful when studying the hippocampus where different cell types are precisely arranged across layers and oscillatory activity is particularly prominent; these markers can be applied to the neocortex as well, to some extent. Recently, other electrophysiological processes have been associated with inhibitory activity and open new avenues for the monitoring of inhibition.

\section{Anatomical distribution}

The anatomical location of recorded spikes is a vital source of information when identifying cell type. In the neocortex, the various types of interneurons are not evenly distributed across layers. PV-positive neurons are more densely located in layer 4, while VIP neurons are more densely located in layers 2/3 (Prönneke et al., 2015) and SST in layers 6/5 (Tremblay et al., 2016).

Similar inhomogeneous distribution is observed in the hippocampus (Freund and Buzsáki, 1996; Harris et al., 2018). Quite obviously, the excitatory cells are almost exclusively located in the pyramidal layer. As a result, any action potential detected outside the pyramidal layer must be emitted by an inhibitory cell (Csicsvari et al., 1999). Neurons of the stratum alveus/oriens (at the level of the basal dendrites of pyramidal cells) are mostly SST-positive interneurons (Freund and Buzsáki, 1996; Harris et al., 2018).

\section{Oscillatory modulation}

Modulation by intrinsic oscillatory patterns is another important marker of cell identity (Csicsvari et al., 1999; Royer et al., 2012; Somogyi and Klausberger, 2005). During locomotion and REM sleep, the hippocampus is dominated by theta oscillations (6-9 Hz), especially in rodents (Buzsáki, 2002). SST neurons in the stratum oriens show slightly delayed phase preference in theta waves relative to PV neurons of the pyramidal layer which fire at the trough of the oscillation (Csicsvari et al., 1999; Royer et al., 2012). Modulation by the hippocampal sharp-waves ripples events (which occurs during immobility and non-REM sleep, Buzsáki, 2015) differs between cell types even more than theta modulation, with the PV neurons of the pyramidal layer being the most responsive to this oscillation (Royer et al., 2012). The modulation of other inhibitory neurons by sharp-waves is more variable, as different subclasses show sometimes opposite response as, for example, bistratified 
and OLM (both SST positive) (Somogyi and Klausberger, 2005). In addition, the positive modulation of PV-positive neurons may be restricted to FS neurons as other PV-positive neurons, such as axo-axonic neurons, show opposite (i.e. negative) modulation during SWRs (Somogyi and Klausberger, 2005). Pyramidal cells also show a broad distribution of modulation by sharp-wave ripples (Royer et al., 2012) possibly because of the differences in circuit integration between superficial and deep layer pyramidal cells (Valero et al., 2015).

In the cortex, the oscillatory entrainment of different neurons is progressively unveiled. For example, FS neurons are strongly entrained by gamma oscillations (30$80 \mathrm{~Hz}$ ) as they actively participate in the generation of these rhythms (Cardin et al., 2009). During sleep, the thalamus generates the so-called spindle oscillations (8$15 \mathrm{~Hz}$ ) that entrain cortical neurons. Cortical FS neurons are strongly recruited, both in overall firing rates and phase coupling (Kandel and Buzsaki, 1997; Peyrache et al., 2011). In contrast, in the deep layers, broad waveform (putative pyramidal) neurons are poorly modulated by spindles, in rate and in phase (Contreras et al., 1997; Peyrache et al., 2011), as recently confirmed with calcium imaging (Seibt et al., 2017) This may be the consequence of the strong feed-forward activation of FS neurons by thalamocortical inputs, therefore exerting shunting inhibition (Contreras et al., 1997). Interestingly, PV-negative inhibitory neurons of unknown type in the medial prefrontal cortex are, like broad-waveform neurons of the deep layers, not entrained by spindle oscillations (Hartwich et al., 2009).

Spindles and SWRs are ubiquitous in mammals and are primarily observed during rest (i.e. are independent of ongoing behavior). They thus constitute interesting oscillatory patterns for validating cell types across species, although further characterization is still necessary.

\section{Waveforms from inhibitory pre-synaptic terminals}

In some cases, spikes from inhibitory neurons can be detected around their presynaptic terminals. These action potentials may be of similar amplitude as spikes detected close to the soma. Such observations were recently made in the thalamus, where the inhibitory neurons of the reticular nucleus are anatomically segregated from the excitatory thalamocortical neurons (Barthó et al., 2014). Barthó and collaborators demonstrated that narrow waveform neurons are sometimes detected in sensory thalamic nuclei where local inhibitory neurons are virtually absent (at least in rodents), thus potentially generated at the pre-synaptic terminals of reticular inhibitory neurons (and resulting from spikes generated at the soma). Interestingly, narrow waveform and broad waveform neurons show different phase preferences to thalamic spindles, therefore confirming that oscillatory entrainment is an important feature in discriminating neuronal types.

\section{Extracellular inhibitory post-synaptic potentials}

In the hippocampus, inhibitory post-synaptic potentials (IPSPs) can be observed in the extracellular medium in vitro (Bazelot et al., 2010; Beyeler et al., 2013; Glickfeld 
et al., 2009). They can be evoked by the discharge of a single inhibitory neuron within its axonal arbor, but are certainly more pronounced when resulting from coordinated actions from inhibitory neurons. The frequency of these events depends on the local connectivity of neurons, both between pyramidal cells and with the local inhibitory neurons. This is certainly why, in vitro, they are much less frequent in the CA1 region than in CA3 (Beyeler et al., 2013). Interestingly, preliminary evidence suggests that these extracellular IPSPs can be observed in vivo (Dubanet et al., 2018). It remains to be confirmed whether these events can be detected in other brain areas or whether this is property specific to the CA3 region.

\begin{tabular}{|c|c|c|c|c|c|}
\hline & & Pyr & PV/FS & SST & VIP \\
\hline \multirow{6}{*}{ 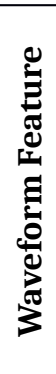 } & Trough-to-peak dur. $(\mu \mathrm{s})^{*, 1}$ & $420-780$ & $170-260$ & $\begin{array}{l}220-540 \\
1,2,+\end{array}$ & $190-610$ \\
\hline & Half-peak dur. $(\mu s)^{*}$ & $320-710$ & $190-300$ & $280-630$ & $370-770$ \\
\hline & Trough-to-peak d' (vs. Pyr) $^{1}$ & & 5.3 & 2.4 & 2.1 \\
\hline & Half-Peak d' (vs. Pyr) $^{1}$ & & 3.6 & 0.6 & 0.4 \\
\hline & Trough-to-peak d' (vs. FS) $^{1}$ & & & 2.9 & 2.5 \\
\hline & Half-Peak d' (vs. FS) $^{1}$ & & & 3 & 4.2 \\
\hline \multirow{4}{*}{ 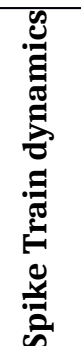 } & Firing rate & Low $(<5 \mathrm{~Hz})$ & $\begin{array}{l}\text { High } \\
>10 \mathrm{~Hz}^{1,2}\end{array}$ & $\begin{array}{l}\text { Broadly } \\
\text { distributed } \\
2,+\end{array}$ & $\begin{array}{l}\text { Interm. } \\
5-15 \mathrm{~Hz}^{3}\end{array}$ \\
\hline & Refractory period & $4-8 \mathrm{~ms}^{4}$ & $<4 \mathrm{~ms}^{4}$ & $2-17 \mathrm{~ms}^{4,+}$ & - \\
\hline & Autocorrelogram mode & $5-15 \mathrm{~ms}^{4}$ & $5-10 \mathrm{~ms}^{4}$ & $10-30 \mathrm{~ms} \mathrm{~s}^{4,+}$ & - \\
\hline & Burst index & $>.5^{4}$ & $0-.5^{4}$ & $<0^{4}$ & - \\
\hline \multirow{3}{*}{ 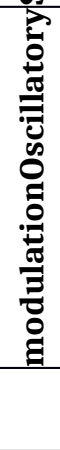 } & $\begin{array}{l}\text { Spindles (PFC, possibly all } \\
\text { neocortex) }\end{array}$ & $\begin{array}{l}\text { L2/3: interm. } \\
\text { L5/6: } \text { low }^{5}\end{array}$ & High & - & - \\
\hline & Sharp-wave ripples (CA1) & $\begin{array}{l}\text { Broadly } \\
\text { distributed }^{1}\end{array}$ & High & $\begin{array}{l}\text { Broadly } \\
\text { distributed } \\
4,+\end{array}$ & - \\
\hline & $\begin{array}{l}\text { Millisecond synchrony with } \\
\text { same cell type? }\end{array}$ & Low $^{6}$ & High $^{2}$ & Low $^{2}$ & \\
\hline \multirow{2}{*}{ 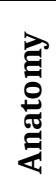 } & $\begin{array}{l}\text { Cortical layers (peak } \\
\text { distribution) }\end{array}$ & & L4 & $\mathrm{L} 5 / 6$ & $\mathrm{~L} 2 / 3$ \\
\hline & CA1 (peak distribution) & Str. pyr. & Str. pyr. & Str. oriens & $\begin{array}{l}\text { Str. } \\
\text { Radiatum }\end{array}$ \\
\hline
\end{tabular}

Table 1 - Properties of the three main inhibitory cell types, relative to pyramidal (pyr) cells. Autocorrelogram mode refers to the time-lag of the first peak in the autocorrelogram. The burst index is computed as the ratio between the number of spikes with inter-spike intervals of less than $10 \mathrm{~ms}$ relative to baseline firing (between 40 and 50ms). Spindle modulation is computed as the percentage of 
neurons whose spikes are significantly phase-locked to the oscillation. Sharp-wave ripple modulation corresponds to the relative change of firing during sharp-waves relative to baseline firing. Inter-neuronal synchrony is evaluated by the relative strength of a central (0-lag) peak in the cross-correlograms.

Data from: ${ }^{1}$, Yu et al. (2019); ${ }^{2}$, Kvitsiani et al. (2013); ${ }^{3}$, Pi et al. (2013); ${ }^{4}$, Royer et al. (2012) ; ${ }^{5}$, Peyrache et al. (2011); ${ }^{6}$, Diba et al. (2014).

, note that waveform features can vary with the filtering parameters of spike detection.

${ }^{+}$, broad distribution possibly because of differences between SST subclasses.

\section{Electrophysiological markers of inhibitory activity are potentially universal}

Electrophysiology remains the only available technique to monitor neuronal activity at single cell resolution across animal species, including in humans implanted with intracerebral electrodes. Although the general organization of the human cortex is similar to other mammalian species, especially in terms of the classes of neurons that constitute the cortical circuits, it remains to be demonstrated that the analytical methods developed to study the electrophysiological markers of neuronal identities in rodents - that constitute the vast majority of the literature - are applicable to both Non-Human Primates (NHPs) and humans. 

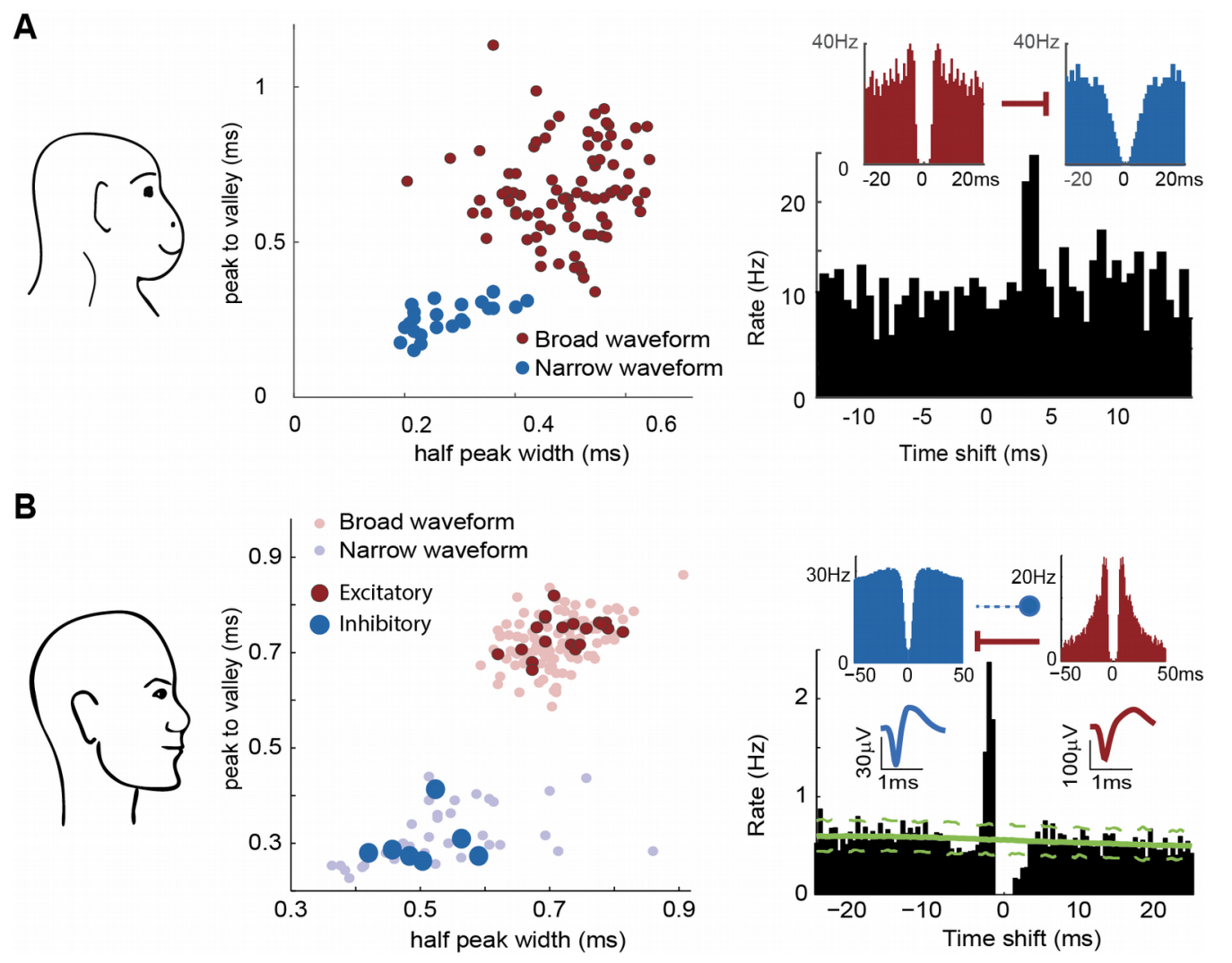

Figure 4 - Cell classification in NHP and human recordings. A. Left, waveform feature classification using half-peak width and trough-to-peak duration, revealing the bimodal distribution of waveform shapes in a narrow (blue dots) and a broad (red dots) waveform clusters. Right, example cross-correlogram showing putative excitatory connection from a broad waveform (reference cell, time 0) to a narrow waveform neuron. Insets display autocorrelograms of the broad (left) and narrow (right) waveform neurons. B. Same as A but from a recording in human epileptic patients. Left, identification of putative synaptic connections shows a perfect match between signs of post-synaptic effect (excitatory or inhibitory) and waveform feature classification. Right, the narrow waveform (FS) neuron is the reference. While the excitatory drive from the broad waveform is highly significant, the dip in the crosscorrelogram, albeit significant, may result from the refractory period of the narrow waveform neuron following excitation by the broad waveform neuron (see text). Note that these neurons were recorded on the same electrode of a Utah array, prevailing the detection of temporally overlapping spikes, thus leading to a 'censored' time around 0. $\boldsymbol{A}$ is adapted from Mendoza et al., 2016; B is adapted from Peyrache et al., 2012.

While electrophysiology in NHPs has a long history, it has long been restricted to the use of single channel electrodes. However, recordings of neuronal ensembles using multi-channel electrode devices are carried out more and more frequently (Dotson et al., 2015; Hoffman, 2002; Mendoza et al., 2016; Schwarz et al., 2014; Smith and Kohn, 2008). These recordings have led to a wealth of data and insights, ranging 
from the organization of neuronal activity in sensory systems, to the development of brain-machine interfaces. In a few cases, differences in waveforms of isolated units led to the demonstration of differential involvement of various cell classes to task and behavioral parameters, e.g. to 'attention' (Mitchell et al., 2007).

Neuronal recordings in humans are quickly becoming standard in neurology and neurosurgery (Babb et al., 1987; Cash and Hochberg, 2015; Engel et al., 2005). This is particularly the case for, but not limited to, patients who suffer from pharmacoresistant epilepsy and thus are candidates for surgery. They can be explored with several intracerebral depth electrodes inserted in various brain regions to localize the epileptic focus, or the source of epileptic seizures. These techniques have led to key discoveries in both clinical research (Cash and Hochberg, 2015) and basic science, for example in the investigation of the neuronal basis of high-level cognitive processes (Ekstrom et al., 2003; Quiroga et al., 2005). Two types of micro-electrodes are available for long-term recordings of single units in the human brain: either a bundle of micro-wires that are extruded at the tip of classical clinical depth electrode probes used to explore the hippocampal formation, or 2D electrode arrays (e.g. Utah Arrays, $4 \mathrm{x} 4 \mathrm{~mm}, 96$ contacts) used to study neuronal activity in the cortical layers (Cash and Hochberg, 2015; Engel et al., 2005). As for NHP research, electrophysiological markers of cell identity were recently explored in various studies. These techniques demonstrated that narrow waveform (putatively inhibitory) neurons and broad waveform (putatively pyramidal) neurons show different entrainment to epileptic patterns (Dehghani et al., 2016; Le Van Quyen et al., 2008; Weiss et al., 2015).

\section{Characterizing cell types in NHPs and humans}

Cell type characterization based on waveform (thus identifying FS cells) has been confirmed to be translatable from rodents to primates (Ison et al., 2011; Le Van Quyen et al., 2008; Mendoza et al., 2016; Peyrache et al., 2012) (Figure 4). When the yield of simultaneously recorded neurons is sufficiently high, it is possible to unravel putative synaptic connections between neurons by analyzing the expected signs of post-synaptic effects based on the waveform classification (Figure 4).

\section{Long recordings are necessary to reveal putative synaptic connections}

Identification of putative connections or millisecond timescale synchrony requires long recordings to collect a sufficient number of spikes and 3-4+ hour recordings in rodents are becoming the norm. To identify significant peaks and troughs in spike train cross-correlograms the temporal resolution must be 1 millisecond at most. For example, to create a cross-correlogram with 1 millisecond bins with at least one event in each bin for neurons firing at approximately $1 \mathrm{~Hz}$, the recording must be at least 1000 seconds long. Thus, for sufficiently high sampling in each bin, recordings may be hours long. In other words, the longer the recordings the better the 
identification of putative synaptic connections (Schwindel et al., 2014). This markedly differs from experimental protocols in which data are only acquired during trials. Recording during sleep, or spontaneous activity, before or after behavioural procedures allows for the collection of data that proves useful when addressing questions such as neuronal connectivity and cell identity. In particular, the matching between BW and FS spike features with the excitatory vs. inhibitory nature of the functional interaction was remarkable for the hours-long human recordings (Peyrache et al., 2012; Fig. 5B), but was not as clear-cut for the shorter NHP recordings (Dehghani et al., 2016). Thus, validating the excitatory or inhibitory nature of recorded neurons, or millisecond synchrony between neurons, an important validation step for cell type identification, requires the acquisition of long recordings, typically of spontaneous activity before or after the task.

\section{Oscillatory markers of cell types.}

As described above, different cell types are differentially entrained to local oscillations in LFPs. Similar observations have now been made in NHPs and humans (Le Van Quyen et al., 2016). For example, gamma oscillations are ubiquitous in the LFPs collected with Utah-array in human epileptic patients across brain states (nonREM sleep, or slow-wave sleep, and wake) (Figure 5A). A small portion of BW cells discharge at higher rates concomitantly with gamma oscillations, while a significant part of FS cells is clearly modulated by the oscillations (Figure 5B). The same result was obtained for beta oscillations in the monkey motor cortex (Le Van Quyen et al., 2016). Although these two oscillation types appear strongly in the LFP, they seem to involve mostly inhibitory FS neurons.

The strong amplitude of gamma oscillations in the LFP presumably results from the extracellular field generated by IPSPs in pyramidal cells (Teleńczuk et al., 2017). However, while pyramidal cell spikes may also contribute to the generation of high frequency oscillations in the LFP (Schomburg et al., 2012), this is not the case with spikes emitted by interneurons (Buzsáki et al., 2012). 

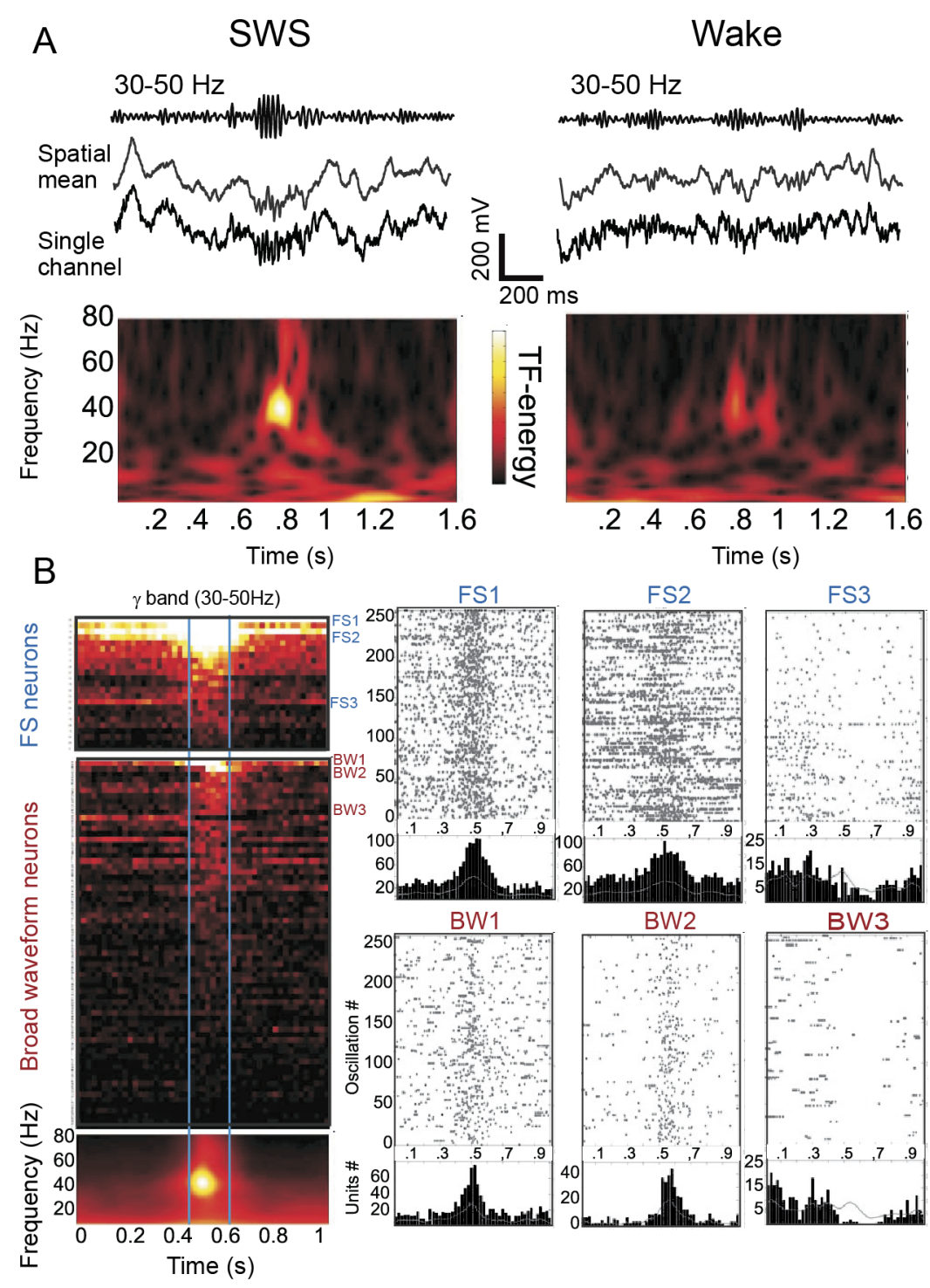

Figure 5 - Gamma oscillations in human and involvement of inhibition. A. Examples of gamma oscillations occurring in slow-wave sleep (SWS) or in wakefulness (Wake) in human temporal cortex. Each panel shows from top to bottom: filtered signal, spatial mean of all LFP signals, one example LFP signal, and time-frequency plots. B. Involvement of inhibition during gamma oscillations. Left: mean modulation of putative inhibitory (FS) and broad waveform cells during gamma episodes. Right: example cells that are modulated by gamma oscillations. In each panel, the cell firing is shown for 250 gamma epochs. Modified with permission from Le Van Quyen et al., 2016.

\section{Properties and role of inhibitory networks}

The role of inhibition in cortical processing and dynamics has been widely studied (for reviews, see Gentet, 2012; Hattori et al., 2017; Kepecs and Fishell, 2014; Roux 
and Buzsáki, 2015; Tremblay et al., 2016; Wood et al., 2017). Here, we address two key aspects of inhibition, which are highly relevant to the study of epilepsy: a local role of inhibition in the generation of action potentials in pyramidal cells and a global role in coordinating activity over large areas of the cortex, with a possible role in the propagation of ictal activity.

\section{Generation of action potentials.}

Spiking of pyramidal cells results from a highly complex integration of synaptic conductance and channel dynamics. Intracellular recordings can reveal the contribution of inhibitory and excitatory conductance to the membrane potentials (Rudolph et al., 2007; Shu et al., 2003). Figure 6A shows an example of conductance measurements from intracellular recordings in the parietal cortex of awake cats (Rudolph et al., 2007). By performing a reverse correlation analysis, it was shown that the total membrane conductance decreases before the spike (Figure 6B). This decrease was caused by a decrease of inhibitory conductance, in other words a disinhibition. Thus, in awake cat parietal cortex, the majority of spikes are not caused by a direct excitation alone but rather by a dis-inhibition (Rudolph et al., 2007). This has important implications for the generation and propagation of epileptiform activity, as sudden shifts in the excitation/inhibition balance is associated with various phases in the transition from interictal regime to the generation of epileptic seizures (Avoli et al., 2016; Lévesque et al., 2016; Weiss et al., 2019).
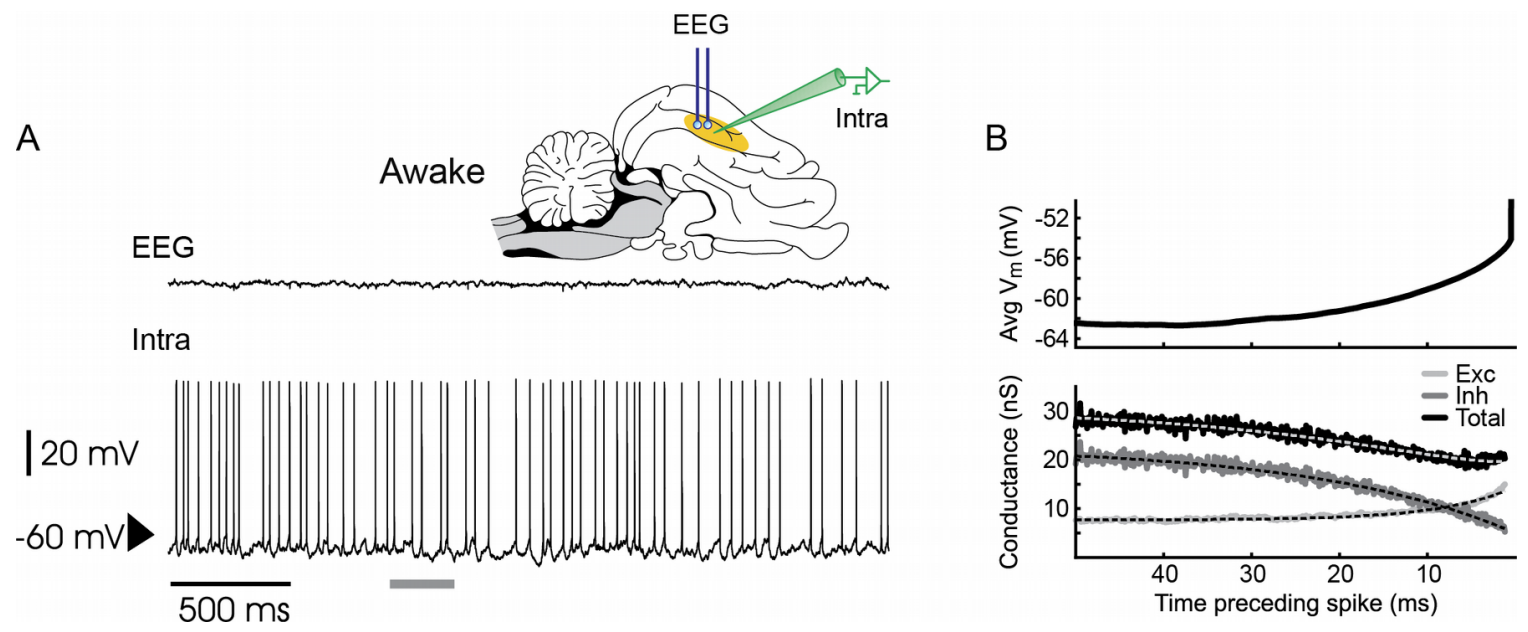

Figure 6 - Disinhibition triggers spikes in awake animals. A. Intracellular recordings in the parietal cortex of awake cats, during desynchronized EEG. The membrane potential was depolarized around $-60 \mathrm{mV}$ and the neurons were tonically firing. $B$. Reverse conductance analysis using the spike-triggered membrane potential (top). The total membrane conductance decreased prior to the spike (black dots); a decrease which was due to the decrease of inhibitory conductance (gray dots). This pattern was observed in 10 out of 11 cells, and was also seen in other brain states. Modified from (Rudolph et al., 2007). 


\section{Long-range coordination of inhibitory activity.}

Cognitive functions emerge from the coordination of vast constellations of neurons distributed in multiple brain areas. Various pathways are believed to underlie cortico-cortical communication, e.g. direct projection between superficial layer pyramidal neurons and relay through high-order thalamic nuclei (Sherman, 2016). Long-range inhibitory networks are another potential candidate for such distributed computation (Buzsaki, 2004), and hippocampal recordings in rats suggest that distal inhibitory cells are more coordinated than pyramidal cells at similar anatomical distance (Hirase et al., 2001).

How can neuronal coordination in space be addressed experimentally? One simple method is to investigate the neuronal pairwise correlation from spatially distributed electrodes. Utah-arrays are ideally suited for this purpose (Kohn and Smith, 2005; Peyrache et al., 2012). They are made of two-dimensional and regularly arranged electrodes (96 in total) with a $400 \mu \mathrm{m}$ pitch. At this distance, putative monosynaptic connections are rarely found, even in long, high quality rodent recordings (English et al., 2014; Fujisawa et al., 2008). However, another pattern in the crosscorrelograms appears at one to two orders of magnitude longer time scales. Certain pairs of neurons display symmetrical 'peaky' cross-correlograms across the duration of hundreds of milliseconds (Figure 7A) that are indicative of coordinated activity at slow timescales. Recording with Utah arrays enables the resolution of the spatial distribution of these correlations (Figure 7B). It can be concluded that in humans, broad waveform neurons, and thus certainly pyramidal cells, are coordinated in ranges of about $1 \mathrm{~mm}$. Interestingly, this corresponds to the typical size of their axonal and dendritic arborization. Conversely, FS neurons are correlated over a much larger spatial extent. No decay with distance could be seen in the spatial range of Utah arrays (maximum $4 \mathrm{~mm}$ ),

The origin of long-range coordination between various FS neurons remains unknown. It could result from common inputs, especially from the thalamus, that would cover larger anatomical areas than for pyramidal cells. These common inputs may not be reflected in the firing of pyramidal cells because of their intrinsically lower excitability and non-linear integration of synaptic inputs.

Although there is no general agreement on the underpinnings of long-range coordination, this observation raises the possibility that an inter-neuronal network potentially plays a key role in the propagation of epileptic activity. Local ictal activity can propagate between cortical regions and subsequently evolve into complex seizure manifestations (Smith et al., 2016). 

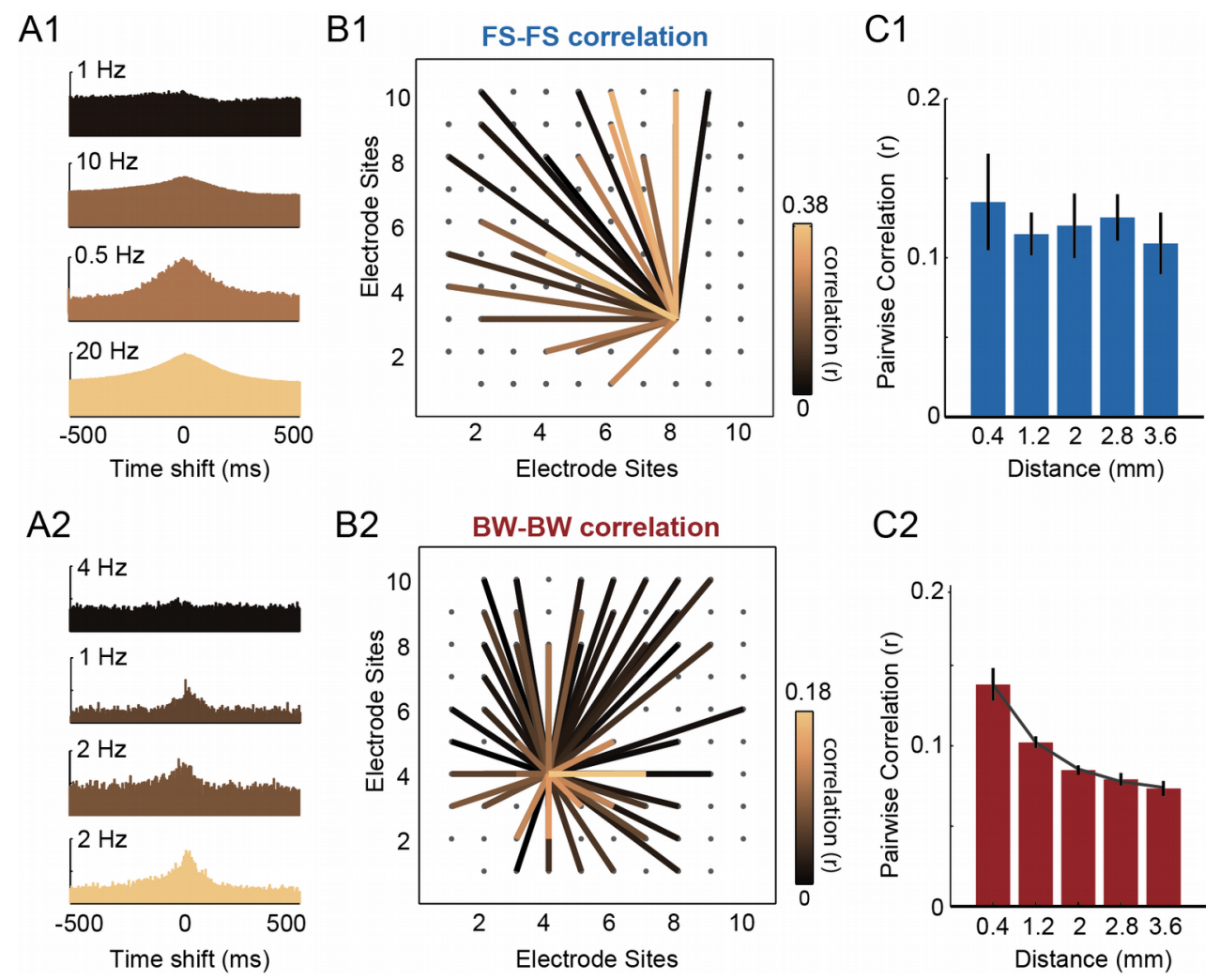

Figure 7 - Putative FS cells are correlated on large spatial extent while correlation among broad waveform neurons decay rapidly with distance. A1. Example crosscorrelograms between one FS neuron and four others. Firing rate of target neurons are indicated on the y-axis. Colors code for correlation value (see B1). B1. Spatial distribution of cell-cell interactions in an example $2 D$ recording session. Left, correlation values of the putative FS (narrow waveform) cell (same as in A1) with all other FS cells simultaneously recorded. Color codes for the absolute value of Pearson's correlation (calculated for 50-ms bins), with black (resp. copper) indicating low (resp. high) correlation values. C1. Normalized correlation coefficients (to account for different basal firing rates) were averaged over 0.8- $\mathrm{mm}$ spatial intervals for all pairs of FS neurons. A2-C2, same as A1-C1 but for broad waveform (BW) neurons. Note that in $C 2, B W-B W$ correlation values are well fitted with an exponential characterized by a decay distance of $\sim 1 \mathrm{~mm}$. Adapted from Peyrache et al., 2012.

\section{Inhibitory activity in epilepsy}

It was long assumed that epileptic activity, either during ictal seizure or interictal spikes, resulted from altered excitatory networks that had become too excitable. However, it is becoming clear that inhibition plays a central role in these processes (see Weiss et al. in the present issue for a comprehensive review on the subject). The development of multi-channel and high-resolution electrophysiology in human epileptic patients has recently allowed to unravel the firing characteristics of 
putative PV/FS and pyramidal neurons during epileptic seizures (Dehghani et al., 2016). While excitation and inhibition usually co-fluctuate in the cortex (Shu et al., 2003), ictal activity is characterized by a strikingly unbalanced regime in which the firing of BW neurons, but not FS, stops (Figure 8). The recording of such ictal events remains challenging as it requires a continuous recording of the micro-electrode, especially for patients who have only a few seizures during the duration of their implantation. In addition, the seizure may lead to mechanical drifts of the electrodes, resulting in erroneous spike detection. At any rate, further recordings of such events, across brain structures, are thus necessary to build a bridge between animal research and observations in human patients.

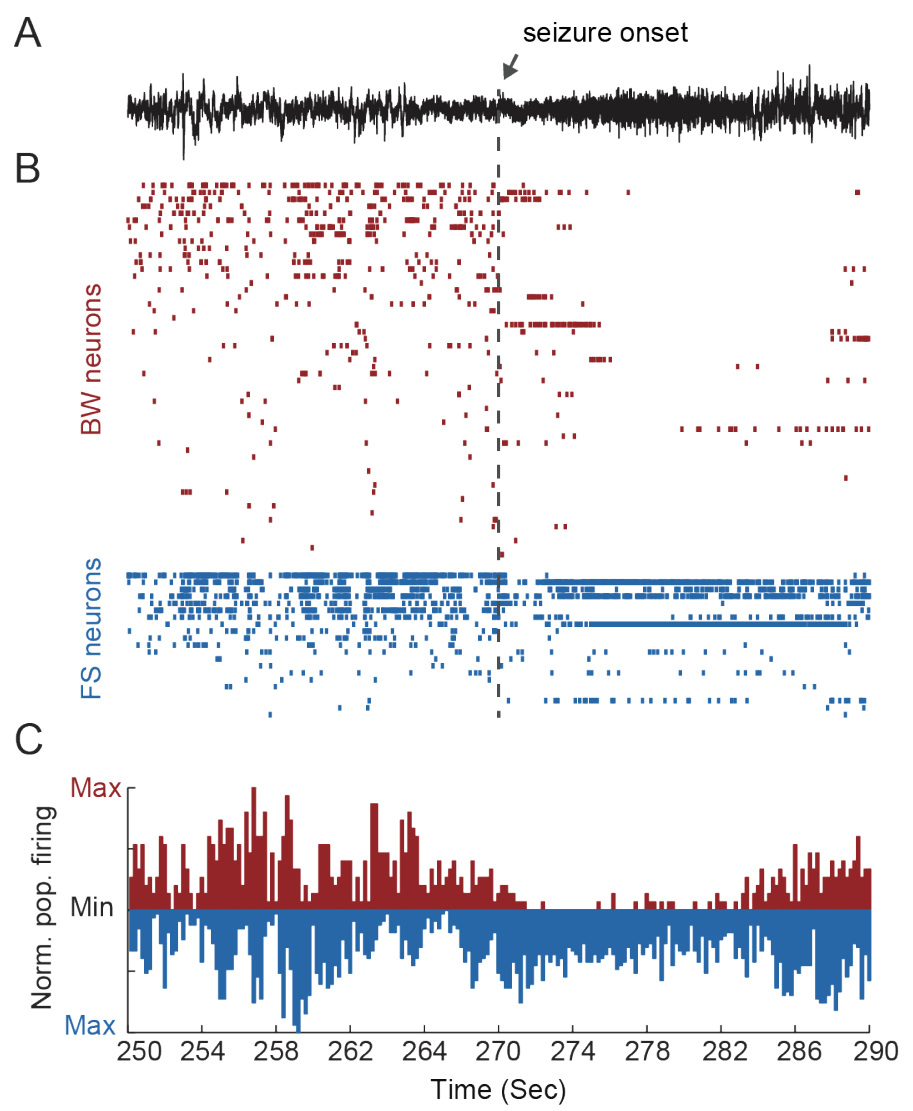

Figure 8-Excitatory/Inhibitory unbalance at the onset of a seizure in human patient, recorded with a multi-channel Utah electrode array. A. Representative LFP trace from one of the microelectrodes.. Arrow indicates seizure onset. B. Rater plot of BW (top, red) and FS (bottom, blue) neurons. $C$. Normalized population firing of $B W$ and FS neurons. Note in $B$ and $C$ the abrupt silencing of BW but not FS neuron spiking.

While electrophysiology offers a unique way to the activity of single neurons and potentially of a specific cell type, at least two techniques have been developed to monitor, non-invasively and indirectly, inhibitory activity in the human brain: Transcranial Magnetic Stimulation (TMS) and Magnetic Resonant Spectroscopy 
(MRS). TMS can activate localized cortical circuits by inducing electric current in a brain area (Barker et al., 1985; Groppa et al., 2012). Specific protocols have been developed that are believed to reveal activation of local inhibitory networks (Kujirai et al., 1993; Lazzaro et al., 1998; McDonnell et al., 2006). Recently, these methods have elegantly confirmed the diminished efficiency of inhibitory neurons in cases of Dravet syndrome (Stern et al., 2017). Inhibition can also be monitored indirectly by tracking the MRS signature of GABA in various brain regions, usually relative to certain metabolites (Puts and Edden, 2012). This technique has revealed changes in GABA concentration during cognitive tasks (Schmitz et al., 2017) and various brain disorders (Harada et al., 2011), but with mixed results in epilepsy (Simister et al., 2003). A combination of these techniques with electrophysiology is a promising lead to further our understanding of inhibitory activity in epilepsy.

\section{Discussion}

In this review, we have presented a state-of-art in classification of neuronal types using extracellular electrophysiology, the only technique able to record single cells in the human brain. This allows for the monitoring of inhibitory activity, especially in the cortex. This is particularly important because inhibitory neurons play a crucial role in the dynamics and operation of cortical circuits. Malfunction in the inhibitory networks likely results in a wide range of neurological disorders (Marín, 2012), from epilepsy (Avoli et al., 2016; Grasse et al., 2013; Lévesque et al., 2016; Neumann et al., 2017) to schizophrenia (Ferrarelli and Tononi, 2011).

Extracellular recordings offer limited, but useful information that, theoretically, allows for the classification of neurons into their various subclasses. These features are twofold: first, single cell features, that include spike waveforms (McCormick et al., 1985), single cell dynamics (Royer et al., 2012) and anatomical location (Freund and Buzsáki, 1996; Harris et al., 2018; Tremblay et al., 2016); second, inter-neuronal interactions in the form of putative synaptic connections (Bartho, 2004; Sirota et al., 2008), spiking millisecond synchrony (Diba et al., 2014; Hu et al., 2011) and oscillatory entrainement (Cardin et al., 2009; Royer et al., 2012). Many of these features have now been explored in human epileptic patients (Dehghani et al., 2016; Ison et al., 2011; Le Van Quyen et al., 2008, 2016; Peyrache et al., 2012; Weiss et al., 2016).

Although neurons show a wide variety of waveforms and firing properties, it is quite surprising that the distinction of cell types using extracellular electrophysiological recordings is mostly limited to FS cells, based on the duration of the waveforms. Currently available data suggest that SST and VIP neurons have intermediate waveform features (Kvitsiani et al., 2013; Pi et al., 2013; Yu et al., 2019) but can potentially be distinguished from FS/PV neurons. Other markers are available for this distinction. For example, SST-positive neurons have longer refractory periods than FS neurons (Royer et al., 2012) and, while FS/PV cells tend to be strongly 
synchronous with each other, SST-positive neurons are not (Kvitsiani et al., 2013). These properties need to be further validated. This will allow one day for the characterization of the activity of non-FS/PV inhibitory neurons in NHPs and human patients.

Identifying inhibitory neurons requires the sampling of large ensembles of neurons. This has been possible for almost two decades in small mammals, with ever progressing techniques (Buzsáki, 2004; Jun et al., 2017). In parallel, the promising development of multi-channel intracerebral recordings in humans opens avenues for the characterization of inhibitory activity in epilepsy patients (Babb et al., 1987; Cash and Hochberg, 2015; Engel et al., 2005). This makes the need for cell class characterization even more pressing.

Epilepsy is certainly the neurological disorder that will benefit the most from the ability to monitor the activity of specific inhibitory cell classes. Understanding the neuronal basis of epileptic discharge at seizure onset and propagation in the brain is a crucial step towards furthering our understanding of the disease. Determining the participation of various cell classes in these pathological neuronal discharges will pave the way to the development of new treatments.

\section{Conclusion}

Future diagnosis of epilepsy (and possibly other neurological disorders) will likely rely on a combination of methods to investigate inhibitory functions, which could enable the identification of malfunctions in specific cortical circuits. The convergence of single cell resolution techniques and markers of inhibitory activity at the scale of entire brain areas will undoubtedly provide a foundation for the understanding of brain dynamics in healthy and pathological conditions.

\section{Acknowledgements}

The authors thank Emily Gittings and Lynda Mainville for carefully editing the manuscript, and Morgane Butler and Melissa Vollrath for the drawings of the monkey and human faces (Figure 4). This work was supported by a Canadian Research Chair in Systems Neuroscience (245716), CIHR Project Grant (155957), NSERC Discovery Grant (RGPIN-2018-04600) and IRDC (108877-001) (A.P.), and by the CNRS and the European Community Future and Emerging Technologies program (Human Brain Project, grant H2020-785907) (A.D.).

\section{References}


Ährlund-Richter, S., Xuan, Y., Lunteren, J.A. van, Kim, H., Ortiz, C., Dorocic, I.P., Meletis, K., and Carlén, M. (2019). A whole-brain atlas of monosynaptic input targeting four different cell types in the medial prefrontal cortex of the mouse. Nat. Neurosci. 22, 657.

Alonso, J.-M., and Swadlow, H.A. (2005). Thalamocortical Specificity and the Synthesis of Sensory Cortical Receptive Fields. J. Neurophysiol. 94, 26-32.

Amarasingham, A., Harrison, M.T., Hatsopoulos, N.G., and Geman, S. (2011). Conditional modeling and the jitter method of spike resampling. J. Neurophysiol. 107, 517-531.

Anastassiou, C.A., Perin, R., Buzsáki, G., Markram, H., and Koch, C. (2015). Cell typeand activity-dependent extracellular correlates of intracellular spiking. J. Neurophysiol. 114, 608-623.

Avoli, M., de Curtis, M., Gnatkovsky, V., Gotman, J., Köhling, R., Lévesque, M., Manseau, F., Shiri, Z., and Williams, S. (2016). Specific imbalance of excitatory/inhibitory signaling establishes seizure onset pattern in temporal lobe epilepsy. J. Neurophysiol. 115, 3229-3237.

Babb, T.L., Wilson, C.L., and Isokawa-Akesson, M. (1987). Firing patterns of human limbic neurons during stereoencephalography (SEEG) and clinical temporal lobe seizures. Electroencephalogr. Clin. Neurophysiol. 66, 467-482.

Barker, A.T., Jalinous, R., and Freeston, I.L. (1985). Non-invasive magnetic stimulation of human motor cortex. Lancet Lond. Engl. 1, 1106-1107.

Bartho, P. (2004). Characterization of Neocortical Principal Cells and Interneurons by Network Interactions and Extracellular Features. J. Neurophysiol. 92, 600-608.

Barthó, P., Slézia, A., Mátyás, F., Faradzs-Zade, L., Ulbert, I., Harris, K.D., and Acsády, L. (2014). Ongoing Network State Controls the Length of Sleep Spindles via Inhibitory Activity. Neuron 82, 1367-1379.

Bazelot, M., Dinocourt, C., Cohen, I., and Miles, R. (2010). Unitary inhibitory field potentials in the CA3 region of rat hippocampus. J. Physiol. 588, 2077-2090.

Bédard, C., Kröger, H., and Destexhe, A. (2004). Modeling Extracellular Field Potentials and the Frequency-Filtering Properties of Extracellular Space. Biophys. J. $86,1829-1842$.

Beyeler, A., Retailleau, A., Molter, C., Mehidi, A., Szabadics, J., and Leinekugel, X. (2013). Recruitment of Perisomatic Inhibition during Spontaneous Hippocampal Activity In Vitro. PLOS ONE 8, e66509. 
Borg-Graham, L.J., Monier, C., and Fregnac, Y. (1998). Visual input evokes transient and strong shunting inhibition in visual cortical neurons. Nature 393, 369-372.

Buzsáki, G. (2002). Theta oscillations in the hippocampus. Neuron 33, 325-340.

Buzsaki, G. (2004). Interneuron Diversity series: Circuit complexity and axon wiring economy of cortical interneurons. Trends Neurosci. 27, 186-193.

Buzsáki, G. (2004). Large-scale recording of neuronal ensembles. Nat. Neurosci. 7, 446-451.

Buzsáki, G. (2015). Hippocampal sharp wave-ripple: A cognitive biomarker for episodic memory and planning. Hippocampus 25, 1073-1188.

Buzsaki, G., and Draguhn, A. (2004). Neuronal oscillations in cortical networks. Science 304, 1926.

Buzsáki, G., and Watson, B.O. (2012). Brain rhythms and neural syntax: implications for efficient coding of cognitive content and neuropsychiatric disease. Dialogues Clin. Neurosci. 14, 345-367.

Buzsáki, G., Anastassiou, C.A., and Koch, C. (2012). The origin of extracellular fields and currents--EEG, ECoG, LFP and spikes. Nat. Rev. Neurosci. 13, 407-420.

Cardin, J.A., Carlén, M., Meletis, K., Knoblich, U., Zhang, F., Deisseroth, K., Tsai, L.-H., and Moore, C.I. (2009). Driving fast-spiking cells induces gamma rhythm and controls sensory responses. Nature 459, 663.

Cash, S.S., and Hochberg, L.R. (2015). The Emergence of Single Neurons in Clinical Neurology. Neuron 86, 79-91.

Connors, B.W., and Gutnick, M.J. (1990). Intrinsic firing patterns of diverse neocortical neurons. Trends Neurosci. 13, 99-104.

Contreras, D., Destexhe, A., and Steriade, M. (1997). Intracellular and computational characterization of the intracortical inhibitory control of synchronized thalamic inputs in vivo. J. Neurophysiol. 78, 335.

Cruikshank, S.J., Lewis, T.J., and Connors, B.W. (2007). Synaptic basis for intense thalamocortical activation of feedforward inhibitory cells in neocortex. Nat. Neurosci. 10, 462-468.

Cruikshank, S.J., Urabe, H., Nurmikko, A.V., and Connors, B.W. (2010). PathwaySpecific Feedforward Circuits between Thalamus and Neocortex Revealed by Selective Optical Stimulation of Axons. Neuron 65, 230-245. 
Csicsvari, J., Hirase, H., Czurko, A., Mamiya, A., and Buzsaki, G. (1999). Oscillatory coupling of hippocampal pyramidal cells and interneurons in the behaving rat. J. Neurosci. 19, 274.

Dehghani, N., Peyrache, A., Telenczuk, B., Le Van Quyen, M., Halgren, E., Cash, S.S., Hatsopoulos, N.G., and Destexhe, A. (2016). Dynamic Balance of Excitation and Inhibition in Human and Monkey Neocortex. Sci. Rep. 6, 23176.

Diba, K., Amarasingham, A., Mizuseki, K., and Buzsáki, G. (2014). Millisecond Timescale Synchrony among Hippocampal Neurons. J. Neurosci. 34, 14984-14994.

Dotson, N.M., Goodell, B., Salazar, R.F., Hoffman, S.J., and Gray, C.M. (2015). Methods, caveats and the future of large-scale microelectrode recordings in the non-human primate. Front. Syst. Neurosci. 9.

Dubanet, O., Beyeler, A., Lecorf, K., Frick, A., Hirase, H., and Leinekugel, X. (2018). Probing the polarity of perisomatic GABAergic transmission in the adult mouse hippocampus in vivo. Program No 732052018 Neurosci. Meet. Plan. San Diego CA Soc. Neurosci. 2018.

Ekstrom, A.D., Kahana, M.J., Caplan, J.B., Fields, T.A., Isham, E.A., Newman, E.L., and Fried, I. (2003). Cellular networks underlying human spatial navigation. Nature 425, 184-188.

Engel, A.K., Moll, C.K.E., Fried, I., and Ojemann, G.A. (2005). Invasive recordings from the human brain: clinical insights and beyond. Nat. Rev. Neurosci. 6, 35-47.

English, D.F., Peyrache, A., Stark, E., Roux, L., Vallentin, D., Long, M.A., and Buzsáki, G. (2014). Excitation and inhibition compete to control spiking during hippocampal ripples: intracellular study in behaving mice. J. Neurosci. Off. J. Soc. Neurosci. 34, 16509-16517.

English, D.F., McKenzie, S., Evans, T., Kim, K., Yoon, E., and Buzsáki, G. (2017). Pyramidal Cell-Interneuron Circuit Architecture and Dynamics in Hippocampal Networks. Neuron 96, 505-520.e7.

Ferrarelli, F., and Tononi, G. (2011). The thalamic reticular nucleus and schizophrenia. Schizophr. Bull. 37, 306-315.

Ferrarelli, F., Huber, R., Peterson, M.J., Massimini, M., Murphy, M., Riedner, B.A., Watson, A., Bria, P., and Tononi, G. (2007). Reduced Sleep Spindle Activity in Schizophrenia Patients. Am. J. Psychiatry 164, 483-492.

Fitzgerald, P.J., and Watson, B.O. (2018). Gamma oscillations as a biomarker for major depression: an emerging topic. Transl. Psychiatry 8, 177. 
Freund, T.F., and Buzsáki, G. (1996). Interneurons of the hippocampus. Hippocampus $6,347-470$.

Fries, P. (2005). A mechanism for cognitive dynamics: neuronal communication through neuronal coherence. Trends Cogn. Sci. 9, 474-480.

Fujisawa, S., Amarasingham, A., Harrison, M.T., and Buzsáki, G. (2008). Behaviordependent short-term assembly dynamics in the medial prefrontal cortex. Nat. Neurosci. 11, 823-833.

Gabernet, L., Jadhav, S.P., Feldman, D.E., Carandini, M., and Scanziani, M. (2005). Somatosensory Integration Controlled by Dynamic Thalamocortical Feed-Forward Inhibition. Neuron 48, 315-327.

Galarreta, M., Erdélyi, F., Szabó, G., and Hestrin, S. (2004). Electrical Coupling among Irregular-Spiking GABAergic Interneurons Expressing Cannabinoid Receptors. J. Neurosci. 24, 9770-9778.

Gentet, L.J. (2012). Functional diversity of supragranular GABAergic neurons in the barrel cortex. Front. Neural Circuits 6.

Gentet, L.J., Avermann, M., Matyas, F., Staiger, J.F., and Petersen, C.C.H. (2010). Membrane Potential Dynamics of GABAergic Neurons in the Barrel Cortex of Behaving Mice. Neuron 65, 422-435.

Gibson, J.R., Beierlein, M., and Connors, B.W. (1999). Two networks of electrically coupled inhibitory neurons in neocortex. Nature 402, 75-79.

Glickfeld, L.L., Roberts, J.D., Somogyi, P., and Scanziani, M. (2009). Interneurons hyperpolarize pyramidal cells along their entire somatodendritic axis. Nat. Neurosci. $12,21-23$.

Gold, C. (2006). On the Origin of the Extracellular Action Potential Waveform: A Modeling Study. J. Neurophysiol. 95, 3113-3128.

Gold, C., Henze, D.A., Koch, C., and Buzsáki, G. (2006). On the Origin of the Extracellular Action Potential Waveform: A Modeling Study. J. Neurophysiol. 95, 3113-3128.

Goldberg, E.M., and Coulter, D.A. (2013). Mechanisms of epileptogenesis: a convergence on neural circuit dysfunction. Nat. Rev. Neurosci. 14, 337.

Grasse, D.W., Karunakaran, S., and Moxon, K.A. (2013). Neuronal synchrony and the transition to spontaneous seizures. Exp. Neurol. 248, 72-84.

Groppa, S., Oliviero, A., Eisen, A., Quartarone, A., Cohen, L.G., Mall, V., Kaelin-Lang, A., Mima, T., Rossi, S., Thickbroom, G.W., et al. (2012). A practical guide to diagnostic 
transcranial magnetic stimulation: Report of an IFCN committee. Clin. Neurophysiol. $123,858-882$.

Harada, M., Taki, M.M., Nose, A., Kubo, H., Mori, K., Nishitani, H., and Matsuda, T. (2011). Non-invasive evaluation of the GABAergic/glutamatergic system in autistic patients observed by MEGA-editing proton MR spectroscopy using a clinical 3 tesla instrument. J. Autism Dev. Disord. 41, 447-454.

Harris, K.D., Henze, D.A., Csicsvari, J., Hirase, H., and Buzsáki, G. (2000). Accuracy of tetrode spike separation as determined by simultaneous intracellular and extracellular measurements. J. Neurophysiol. 84, 401-414.

Harris, K.D., Hochgerner, H., Skene, N.G., Magno, L., Katona, L., Gonzales, C.B., Somogyi, P., Kessaris, N., Linnarsson, S., and Hjerling-Leffler, J. (2018). Classes and continua of hippocampal CA1 inhibitory neurons revealed by single-cell transcriptomics. PLOS Biol. 16, e2006387.

Hartwich, K., Pollak, T., and Klausberger, T. (2009). Distinct firing patterns of identified basket and dendrite-targeting interneurons in the prefrontal cortex during hippocampal theta and local spindle oscillations. J. Neurosci. 29, 9563.

Hattori, R., Kuchibhotla, K.V., Froemke, R.C., and Komiyama, T. (2017). Functions and dysfunctions of neocortical inhibitory neuron subtypes. Nat. Neurosci. 20, 11991208.

Hirase, H., Leinekugel, X., Csicsvari, J., Czurko, A., and Buzsaki, G. (2001). Behaviordependent states of the hippocampal network affect functional clustering of neurons. J. Neurosci. 21, 145.

Hoffman, K.L. (2002). Coordinated Reactivation of Distributed Memory Traces in Primate Neocortex. Science 297, 2070-2073.

Hu, H., Ma, Y., and Agmon, A. (2011). Submillisecond Firing Synchrony between Different Subtypes of Cortical Interneurons Connected Chemically But Not Electrically. J. Neurosci. 31, 3351-3361.

Isaacson, J.S., and Scanziani, M. (2011). How Inhibition Shapes Cortical Activity. Neuron 72, 231-243.

Ison, M.J., Mormann, F., Cerf, M., Koch, C., Fried, I., and Quiroga, R.Q. (2011).

Selectivity of pyramidal cells and interneurons in the human medial temporal lobe. J. Neurophysiol. 106, 1713-1721.

Jun, J.J., Steinmetz, N.A., Siegle, J.H., Denman, D.J., Bauza, M., Barbarits, B., Lee, A.K., Anastassiou, C.A., Andrei, A., Aydın, Ç., et al. (2017). Fully integrated silicon probes for high-density recording of neural activity. Nature 551, 232-236. 
Kandel, A., and Buzsaki, G. (1997). Cellular-synaptic generation of sleep spindles, spike-and-wave discharges, and evoked thalamocortical responses in the neocortex of the rat. J. Neurosci. 17, 6783.

Kepecs, A., and Fishell, G. (2014). Interneuron cell types are fit to function. Nature $505,318-326$.

Kerlin, A.M., Andermann, M.L., Berezovskii, V.K., and Reid, R.C. (2010). Broadly Tuned Response Properties of Diverse Inhibitory Neuron Subtypes in Mouse Visual Cortex. Neuron 67, 858-871.

Kohn, A., and Smith, M.A. (2005). Stimulus Dependence of Neuronal Correlation in Primary Visual Cortex of the Macaque. J. Neurosci. 25, 3661-3673.

Kujirai, T., Caramia, M.D., Rothwell, J.C., Day, B.L., Thompson, P.D., Ferbert, A., Wroe, S., Asselman, P., and Marsden, C.D. (1993). Corticocortical inhibition in human motor cortex. J. Physiol. 471, 501-519.

Kvitsiani, D., Ranade, S., Hangya, B., Taniguchi, H., Huang, J.Z., and Kepecs, A. (2013). Distinct behavioural and network correlates of two interneuron types in prefrontal cortex. Nature 498, 363-366.

Lazzaro, V.D., Restuccia, D., Oliviero, A., Profice, P., Ferrara, L., Insola, A., Mazzone, P., Tonali, P., and Rothwell, J.C. (1998). Magnetic transcranial stimulation at intensities below active motor threshold activates intracortical inhibitory circuits. Exp. Brain Res. 119, 265-268.

Le Van Quyen, M., Bragin, A., Staba, R., Crépon, B., Wilson, C.L., and Engel, J. (2008). Cell Type-Specific Firing during Ripple Oscillations in the Hippocampal Formation of Humans. J. Neurosci. 28, 6104-6110.

Le Van Quyen, M., Muller, L.E., Telenczuk, B., Halgren, E., Cash, S., Hatsopoulos, N.G., Dehghani, N., and Destexhe, A. (2016). High-frequency oscillations in human and monkey neocortex during the wake-sleep cycle. Proc. Natl. Acad. Sci. 113, 93639368.

Lefebvre, B., Yger, P., and Marre, O. (2016). Recent progress in multi-electrode spike sorting methods. J. Physiol. Paris 110, 327-335.

Lévesque, M., Herrington, R., Hamidi, S., and Avoli, M. (2016). Interneurons spark seizure-like activity in the entorhinal cortex. Neurobiol. Dis. 87, 91-101.

Marín, O. (2012). Interneuron dysfunction in psychiatric disorders. Nat. Rev. Neurosci. 13, 107-120. 
McCormick, D.A., Connors, B.W., Lighthall, J.W., and Prince, D.A. (1985). Comparative electrophysiology of pyramidal and sparsely spiny stellate neurons of the neocortex. J. Neurophysiol. 54, 782-806.

McDonnell, M.N., Orekhov, Y., and Ziemann, U. (2006). The role of GABAB receptors in intracortical inhibition in the human motor cortex. Exp. Brain Res. 173, 86-93.

Medrihan, L., Sagi, Y., Inde, Z., Krupa, O., Daniels, C., Peyrache, A., and Greengard, P. (2017). Initiation of Behavioral Response to Antidepressants by Cholecystokinin Neurons of the Dentate Gyrus. Neuron 95, 564-576.e4.

Mendoza, G., Peyrache, A., Gámez, J., Prado, L., Buzsáki, G., and Merchant, H. (2016). Recording extracellular neural activity in the behaving monkey using a semichronic and high-density electrode system. J. Neurophysiol. 116, 563-574.

Mitchell, J.F., Sundberg, K.A., and Reynolds, J.H. (2007). Differential AttentionDependent Response Modulation across Cell Classes in Macaque Visual Area V4. Neuron 55, 131-141.

Moore, G.P., Segundo, J.P., Perkel, D.H., and Levitan, H. (1970). Statistical Signs of Synaptic Interaction in Neurons. Biophys. J. 10, 876-900.

Nassar, M., Simonnet, J., Huang, L.-W., Mathon, B., Cohen, I., Bendels, M.H.K., Beraneck, M., Miles, R., and Fricker, D. (2018). Anterior Thalamic Excitation and Feedforward Inhibition of Presubicular Neurons Projecting to Medial Entorhinal Cortex. J. Neurosci. 38, 6411-6425.

Neumann, A.R., Raedt, R., Steenland, H.W., Sprengers, M., Bzymek, K., Navratilova, Z., Mesina, L., Xie, J., Lapointe, V., Kloosterman, F., et al. (2017). Involvement of fastspiking cells in ictal sequences during spontaneous seizures in rats with chronic temporal lobe epilepsy. Brain 140, 2355-2369.

Ostojic, S., Brunel, N., and Hakim, V. (2009). How connectivity, background activity, and synaptic properties shape the cross-correlation between spike trains. J. Neurosci. Off. J. Soc. Neurosci. 29, 10234-10253.

Pachitariu, M., Steinmetz, N., Kadir, S., Carandini, M., and Harris, K.D. (2016). Kilosort: realtime spike-sorting for extracellular electrophysiology with hundreds of channels. bioRxiv 61481.

Perkel, D.H., Gerstein, G.L., and Moore, G.P. (1967). Neuronal spike trains and stochastic point processes. II. Simultaneous spike trains. Biophys. J. 7, 419-440.

Peyrache, A., Battaglia, F.P., and Destexhe, A. (2011). Inhibition recruitment in prefrontal cortex during sleep spindles and gating of hippocampal inputs. Proc. Natl. Acad. Sci. 108, 17207-17212. 
Peyrache, A., Dehghani, N., Eskandar, E.N., Madsen, J.R., Anderson, W.S., Donoghue, J.A., Hochberg, L.R., Halgren, E., Cash, S.S., and Destexhe, A. (2012). Spatiotemporal dynamics of neocortical excitation and inhibition during human sleep. Proc. Natl. Acad. Sci. U. S. A. 109, 1731-1736.

Peyrache, A., Lacroix, M.M., Petersen, P.C., and Buzsáki, G. (2015). Internally organized mechanisms of the head direction sense. Nat. Neurosci. 18, 569-575.

Pi, H.-J., Hangya, B., Kvitsiani, D., Sanders, J.I., Huang, Z.J., and Kepecs, A. (2013). Cortical interneurons that specialize in disinhibitory control. Nature 503, 521-524.

Pinto, L., and Dan, Y. (2015). Cell-Type-Specific Activity in Prefrontal Cortex during Goal-Directed Behavior. Neuron 87, 437-450.

Prönneke, A., Scheuer, B., Wagener, R.J., Möck, M., Witte, M., and Staiger, J.F. (2015). Characterizing VIP Neurons in the Barrel Cortex of VIPcre/tdTomato Mice Reveals Layer-Specific Differences. Cereb. Cortex 25, 4854-4868.

Puts, N.A.J., and Edden, R.A.E. (2012). In vivo magnetic resonance spectroscopy of GABA: A methodological review. Prog. Nucl. Magn. Reson. Spectrosc. 60, 29-41.

Quian Quiroga, R. (2009). What is the real shape of extracellular spikes? J. Neurosci. Methods 177, 194-198.

Quiroga, R.Q., Reddy, L., Kreiman, G., Koch, C., and Fried, I. (2005). Invariant visual representation by single neurons in the human brain. Nature 435, 1102-1107.

Roux, L., and Buzsáki, G. (2015). Tasks for inhibitory interneurons in intact brain circuits. Neuropharmacology 88, 10-23.

Royer, S., Zemelman, B.V., Losonczy, A., Kim, J., Chance, F., Magee, J.C., and Buzsáki, G. (2012). Control of timing, rate and bursts of hippocampal place cells by dendritic and somatic inhibition. Nat. Neurosci. 15, 769-775.

Rudolph, M., Pospischil, M., Timofeev, I., and Destexhe, A. (2007). Inhibition Determines Membrane Potential Dynamics and Controls Action Potential Generation in Awake and Sleeping Cat Cortex. J. Neurosci. 27, 5280-5290.

Rudy, B., Fishell, G., Lee, S., and Hjerling-Leffler, J. (2011). Three groups of interneurons account for nearly $100 \%$ of neocortical GABAergic neurons. Dev. Neurobiol. 71, 45-61.

Schmitz, T.W., Correia, M.M., Ferreira, C.S., Prescot, A.P., and Anderson, M.C. (2017). Hippocampal GABA enables inhibitory control over unwanted thoughts. Nat. Commun. 8, 1311. 
Schomburg, E.W., Anastassiou, C.A., Buzsáki, G., and Koch, C. (2012). The Spiking Component of Oscillatory Extracellular Potentials in the Rat Hippocampus. J. Neurosci. 32, 11798-11811.

Schwarz, D.A., Lebedev, M.A., Hanson, T.L., Dimitrov, D.F., Lehew, G., Meloy, J., Rajangam, S., Subramanian, V., Ifft, P.J., Li, Z., et al. (2014). Chronic, wireless recordings of large-scale brain activity in freely moving rhesus monkeys. Nat. Methods 11, 670-676.

Schwindel, C.D., Ali, K., McNaughton, B.L., and Tatsuno, M. (2014). Long-Term Recordings Improve the Detection of Weak Excitatory-Excitatory Connections in Rat Prefrontal Cortex. J. Neurosci. 34, 5454-5467.

Seibt, J., Richard, C.J., Sigl-Glöckner, J., Takahashi, N., Kaplan, D.I., Doron, G., de Limoges, D., Bocklisch, C., and Larkum, M.E. (2017). Cortical dendritic activity correlates with spindle-rich oscillations during sleep in rodents. Nat. Commun. 8 , 684.

Sherman, S.M. (2016). Thalamus plays a central role in ongoing cortical functioning. Nat. Neurosci. 19, 533-541.

Shu, Y., Hasenstaub, A., and McCormick, D.A. (2003). Turning on and off recurrent balanced cortical activity. Nature 423, 288-293.

Silberberg, G., and Markram, H. (2007). Disynaptic Inhibition between Neocortical Pyramidal Cells Mediated by Martinotti Cells. Neuron 53, 735-746.

Simister, R.J., McLean, M.A., Barker, G.J., and Duncan, J.S. (2003). A Proton Magnetic Resonance Spectroscopy Study of Metabolites in the Occipital Lobes in Epilepsy. Epilepsia 44, 550-558.

Simonnet, J., Nassar, M., Stella, F., Cohen, I., Mathon, B., Boccara, C.N., Miles, R., and Fricker, D. (2017). Activity dependent feedback inhibition may maintain head direction signals in mouse presubiculum. Nat. Commun. 8, 16032.

Sirota, A., Montgomery, S., Fujisawa, S., Isomura, Y., Zugaro, M., and Buzsaki, G. (2008). Entrainment of Neocortical Neurons and Gamma Oscillations by the Hippocampal Theta Rhythm. Neuron 60, 683-697.

Smith, M.A., and Kohn, A. (2008). Spatial and Temporal Scales of Neuronal Correlation in Primary Visual Cortex. J. Neurosci. 28, 12591-12603.

Smith, E.H., Liou, J., Davis, T.S., Merricks, E.M., Kellis, S.S., Weiss, S.A., Greger, B., House, P.A., Ii, G.M.M., Goodman, R.R., et al. (2016). The ictal wavefront is the spatiotemporal source of discharges during spontaneous human seizures. Nat. Commun. 7, 11098. 
Somogyi, P., and Klausberger, T. (2005). Defined types of cortical interneurone structure space and spike timing in the hippocampus. J. Physiol. 562, 9-26.

Stark, E., and Abeles, M. (2009). Unbiased estimation of precise temporal correlations between spike trains. J. Neurosci. Methods 179, 90-100.

Stark, E., Eichler, R., Roux, L., Fujisawa, S., Rotstein, H.G., and Buzsáki, G. (2013). Inhibition-induced theta resonance in cortical circuits. Neuron 80, 1263-1276.

Stern, W.M., Sander, J.W., Rothwell, J.C., and Sisodiya, S.M. (2017). Impaired intracortical inhibition demonstrated in vivo in people with Dravet syndrome. Neurology 88, 1659-1665.

Tamás, G., Buhl, E.H., Lörincz, A., and Somogyi, P. (2000). Proximally targeted GABAergic synapses and gap junctions synchronize cortical interneurons. Nat. Neurosci. 3, 366-371.

Taniguchi, H., He, M., Wu, P., Kim, S., Paik, R., Sugino, K., Kvitsani, D., Fu, Y., Lu, J., Lin, Y., et al. (2011). A Resource of Cre Driver Lines for Genetic Targeting of GABAergic Neurons in Cerebral Cortex. Neuron 71, 995-1013.

Tasic, B., Yao, Z., Graybuck, L.T., Smith, K.A., Nguyen, T.N., Bertagnolli, D., Goldy, J., Garren, E., Economo, M.N., Viswanathan, S., et al. (2018). Shared and distinct transcriptomic cell types across neocortical areas. Nature 563, 72.

Teleńczuk, B., Dehghani, N., Le Van Quyen, M., Cash, S.S., Halgren, E., Hatsopoulos, N.G., and Destexhe, A. (2017). Local field potentials primarily reflect inhibitory neuron activity in human and monkey cortex. Sci. Rep. 7, 40211.

Traub, R.D., Kopell, N., Bibbig, A., Buhl, E.H., LeBeau, F.E.N., and Whittington, M.A. (2001). Gap Junctions between Interneuron Dendrites Can Enhance Synchrony of Gamma Oscillations in Distributed Networks. J. Neurosci. 21, 9478-9486.

Tremblay, R., Lee, S., and Rudy, B. (2016). GABAergic Interneurons in the Neocortex: From Cellular Properties to Circuits. Neuron 91, 260-292.

Valero, M., Cid, E., Averkin, R.G., Aguilar, J., Sanchez-Aguilera, A., Viney, T.J., GomezDominguez, D., Bellistri, E., and de la Prida, L.M. (2015). Determinants of different deep and superficial CA1 pyramidal cell dynamics during sharp-wave ripples. Nat. Neurosci. advance online publication.

Wamsley, B., and Fishell, G. (2017). Genetic and activity-dependent mechanisms underlying interneuron diversity. Nat. Rev. Neurosci. 18, 299.

Wehr, M., and Zador, A.M. (2003). Balanced inhibition underlies tuning and sharpens spike timing in auditory cortex. Nature $426,442-446$. 
Weiss, S.A., Staba, R., Bragin, A., Moxon, K., Sperling, M., Avoli, M., and Engel, J. (2019). Interneurons and principal cell firing in human limbic areas at focal seizure onset. Neurobiol. Dis. 124, 183-188.

Weiss, Shennan Aibel, Alvarado-Rojas, Catalina, Bragin, Anatol, Behnke, Eric, Fields Tony, Fried, Itzhak, Engel, Jerome, and Staba, Richard (2015). Ictal onset patterns of local field potentials, high frequency oscillations, and unit activity in human mesial temporal lobe epilepsy. Epilepsia 57, 111-121.

Wilson, N.R., Runyan, C.A., Wang, F.L., and Sur, M. (2012). Division and subtraction by distinct cortical inhibitory networks in vivo. Nature 488, 343-348.

Wood, K.C., Blackwell, J.M., and Geffen, M.N. (2017). Cortical inhibitory interneurons control sensory processing. Curr. Opin. Neurobiol. 46, 200-207.

Xu, X., Roby, K.D., and Callaway, E.M. (2010). Immunochemical characterization of inhibitory mouse cortical neurons: Three chemically distinct classes of inhibitory cells. J. Comp. Neurol. 518, 389-404.

Yu, J., Hu, H., Agmon, A., and Svoboda, K. (2019). Principles Governing the Dynamics of GABAergic Interneurons in the Barrel Cortex. bioRxiv 554949.

Zeng, H., and Sanes, J.R. (2017). Neuronal cell-type classification: challenges, opportunities and the path forward. Nat. Rev. Neurosci. 18, 530-546.

Zijlmans, M., Jiruska, P., Zelmann, R., Leijten, F.S.S., Jefferys, J.G.R., and Gotman, J. (2012). High-frequency oscillations as a new biomarker in epilepsy. Ann. Neurol. 71, 169-178. 